\title{
CONVERGENCE OF PRICE PROCESSES UNDER TWO DYNAMIC DOUBLE AUCTIONS
}

\author{
Jinpeng Ma \\ Rutgers University, USA \\ jinpeng@camden. rutgers.edu \\ Qiongling Li \\ Rice University, USA \\ ql4arice.edu
}

\begin{abstract}
We study the convergence of two price processes generated by two dynamic double auctions (DA) and provide conditions under which the two price processes converge to a Walrasian equilibrium in the underlying economy. When the conditions are not satisfied, the price processes may result in a bubble or crash.

Keywords: Double auction mechanisms, incremental subgradient methods, network resource allocations.

JEL Classification Numbers: D44, D50.
\end{abstract}

\section{INTRODUCTION}

double auction (DA) mechanism is a market-clearing system by which
dispersed private information feeds into the system sequentially through
bilateral trading. With little concentrated information about total demand and
supply of an asset or good available to all participants in the marketplace, it
Both authors declare there are no conflicts of interest. This paper supersedes the paper
"Bubbles, Crashes and Efficiency with Double Auction Mechanisms" (Ma \& Li, 2011), which
has been distributed and presented in various conferences. We thank Mark Satterthwaite for
introducing us to the topic. Any errors are our own.

Copyright (C) Jinpeng Ma, Qiongling Li / 1(1), 2016, 1-44. 
is natural to ask whether the price process generated by this DA mechanism converges to an equilibrium of the underlying economy or not.

Both A. Smith (1776) and Hayek (1945) raise a similar question how a market mechanism in a laissez-faire economy, where individual participants with little information about total demand and supply act solely in their self-interests, is able to integrate "dispersed bits of [incomplete] information" correctly into prices. A. Smith (1776) uses his famous "invisible hand" metaphor to describe its magnificence of a price mechanism. Hayek (1945, p. 519) has further explored the idea:

"The peculiar character of the problem of a rational economic order is determined precisely by the fact that the knowledge of the circumstances of which we must make use never exists in concentrated or integrated form, but solely as the dispersed bits of incomplete and frequently contradictory knowledge which all the separate individuals possess. The economic problem of society is thus not merely a problem of how to allocate "given" resources $[\cdots]$, it is rather a problem of the utilization of knowledge not given to anyone in its totality."

He goes on by saying: "This mechanism would have been acclaimed as one of the greatest triumphs of the human mind" if "It were the result of deliberate human design" (Hayek, 1945, p. 527). It should be noted that DA mechanisms employed in real exchange markets across the world are deliberately designed by humans.

An answer to the question is important for understanding price determination in an exchange market, since DA mechanisms have been widely used in equity, commodity and currency markets, among others. For example, an answer to the question is vital for understanding the efficient markets hypothesis (Fama, 1965) and the excess volatility puzzle (Shiller, 1981). Nonetheless, it is not easy to come up with an answer. Indeed, does a DA mechanism matter for the price determination of an asset? According to the efficient markets hypothesis, the answer should be no since the price of an asset in an exchange market should always follow its fundamental, with no systematic disparity between the two that can be detected with fundamental or technical analysis. On the other hand, excess volatility suggests that the answer may be yes, since the price of an equity can deviate from its fundamental to a great degree and such a deviation has been realized by a DA mechanism through a sequence of 
trading between buyer and seller pairs. But, if a DA mechanism really matters, how is it possible for an equity with fundamental value of 100 to be traded, say, at 300 or 50 ?

The main objective of this paper is to investigate if a DA mechanism can generate a sequence of prices that converges to an equilibrium of the underlying economy when individual demands and supplies are only privately known. To achieve this goal, we study a benchmark model given below:

$$
\mathscr{P} \quad \operatorname{minimize} F(y)=\sum_{i=1}^{m} f_{i}(y)+\sum_{j=1}^{n} g_{j}(y)
$$

subject to $y \in Y$, a nonempty convex subset of $R_{+}^{d}$, where $f_{i}$ and $g_{j}$ are real-valued (possibly non-differentiable) convex functions defined on the $d$ dimensional Euclidean space $R^{d}$. A large class of quasilinear economies with $m$ sellers and $n$ buyers can be represented by this form (see Section 2.1). For these economies, the quantity demanded and supplied at prices $y$ for buyer $j=1,2, \cdots, n$ and seller $i=1,2, \cdots, m$ are just subsets of the subdifferentials $-\partial g_{j}(y)$ and $\partial f_{i}(y)$, respectively, using the Fenchel duality (Ma \& Nie, 2003). Thus, an equilibrium of the underlying economy studied in this paper is an optimal solution to the problem $\mathscr{P}$.

An Illustrative Example. For simplicity, consider an exchange economy where there is a single object or asset with a finite number of identical copies for sale. In a dynamic double auction, a buyer submits a bid order consisting of a bid price and a bid size, and a seller submits an ask order consisting of an ask price and an ask size, with the bid price at least as high as the ask price. The bid size is the quantity the buyer is willing to buy at the bid price and the ask size is the quantity the seller is willing to sell at the ask price. The price of an object is a weighted average of the bid price and the ask price, with weight $\alpha \in(0,1)$, as in a static double auction in Chatterjee \& Samuelson (1983), Myerson \& Satterthwaite (1983), Wilson (1985), and Gresik (1991). Thus, given a sequence of pairs of one buyer and one seller, a sequence of prices is generated by a double auction. The next two questions are, at a given iteration, who will be the buyer and the seller pair and how are bid and ask prices are determined? We provide two specific examples of double auction to address the two questions.

In the first double auction, we assume that the number of buyers equals the number of sellers, and buyers and sellers form two cyclic rings: a buyer ring and a seller ring (Figure 1). This system can be realized if the buyer and seller 
rings consist of two permutations of agents. A pair of a buyer and a seller is selected according to the two cyclic rings, one pair at a time. The process starts with price $X_{k}$ at $k$. Then buyer $\pi^{\prime}(1)$ and seller $\pi(1)$ are the first pair to submit their bid and ask, respectively, based on the observed $X_{k}$. After the iteration of the pair $\left(\pi^{\prime}(1), \pi(1)\right)$, the next pair will be buyer $\pi^{\prime}(2)$ and seller $\pi(2)$. This iteration process ends with the pair $\left(\pi^{\prime}(m), \pi(m)\right)$ and the price $X_{k+1}$.

Figure 1. Iterations under a double auction, where $\pi$ and $\pi^{\prime}$ are two permutations of agents.
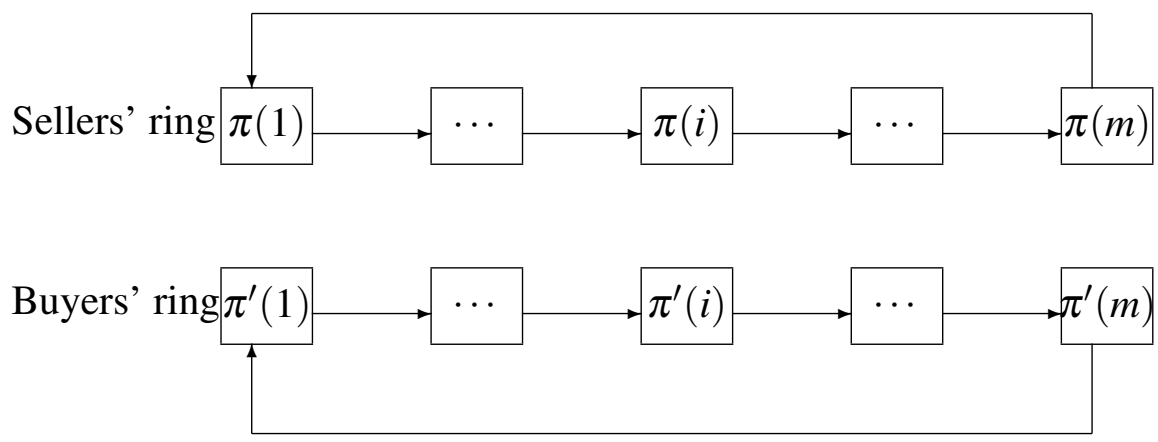

We need to determine how a buyer bids and a seller asks. A buyer's bid equals the newly updated price from the previous pair along the two rings plus a price increment that equals the product of the bid step size and the bid size. The bid step size is the price increment for one unit of the object that the buyer is willing to buy. Thus, the more a buyer wants to buy, the higher the bid price increment. An ask price is determined similarly. A seller's ask price equals the newly updated price from the previous pair along the two rings minus a price that equals the product of the ask step size and the ask size. The ask step size is now the price decrement for one unit of the object for sale. Thus, the more a seller wants to sell, the lower the ask price. To be more precise, let $\Phi_{i-1, k}$ be the price at iteration $k$ and designate the next selected pair as $\left(\pi(i), \pi^{\prime}(i)\right)$. The ask price $\psi_{\pi(i), k}$ and the bid price $\varphi_{\pi^{\prime}(i), k}$ are determined by, respectively,

$$
\psi_{\pi(i), k}=\Phi_{i-1, k}-a_{k} \cdot S_{\pi(i)}\left(\Phi_{i-1, k}\right), \varphi_{\pi^{\prime}(i), k}=\Phi_{i-1, k}+b_{k} \cdot D_{\pi^{\prime}(i)}\left(\Phi_{i-1, k}\right),
$$

where $S_{\pi(i)}\left(\Phi_{i-1, k}\right)$ and $D_{\pi^{\prime}(i)}\left(\Phi_{i-1, k}\right)$ are the quantity supplied (i.e. the ask size) and demanded (i.e. the bid size) at price $\Phi_{i-1, k}$, respectively. If $S_{\pi(i)}\left(\Phi_{i-1, k}\right)$ and $D_{\pi^{\prime}(i)}\left(\Phi_{i-1, k}\right)$ are set-valued maps, equation (1) should be 
understood with two selections from the demand and supply. $\left\{a_{k}\right\}$ and $\left\{b_{k}\right\}$ are the ask and bid step sizes, respectively. The price $\Phi_{i, k}$, which is communicated to the next pair, is determined by a weighted average of the bid and ask prices, with weight $\alpha \in(0,1)$ :

$$
\Phi_{i, k}=\alpha \psi_{\pi(i), k}+(1-\alpha) \varphi_{\pi^{\prime}(i), k} .
$$

Equations (1) and (2) provide the rule on how the price at an iteration evolves from one pair to the other along the two rings. The price process starts at $\Phi_{0, k}=X_{k}$ and ends with $X_{k+1}=\Phi_{m, k}$ at time $k$. Then this process repeats with two different permutations of agents. Thus, a sequence of prices $X_{k}, k=$ $0,1,2, \cdots$, is generated. Note that we consider the case where $m$ is potentially a large number.

In our second randomized double auction a pair made up of a buyer and a seller is independently selected. Here we do not need the condition that the number of buyers equals the number of sellers because such an auction can be seen as a special case, in which the buyer ring and the seller ring in Figure 1 each consist of a single agent. Thus, equations (1) and (2) provide a sequence of prices $\left\{X_{k}\right\}$ once again.

Results. Assume that the underlying economy has a Walrasian equilibrium and the $\operatorname{limit}_{\lim _{k \rightarrow \infty}} \frac{b_{k}}{a_{k}}$ exists for two diminishing step sizes $\left\{a_{k}\right\}$ and $\left\{b_{k}\right\}$. Suppose there is a positive scalar $\lambda$ such that (see Assumption 3.2)

$$
\sum_{k=0}^{\infty}\left|\frac{b_{k}}{n}-\lambda \frac{a_{k}}{m}\right|<+\infty .
$$

Then we show that $\lambda$ must be $\lim _{k \rightarrow \infty} \frac{b_{k}}{a_{k}} \cdot{ }^{1}$ Our first main result Theorem 4.4 demonstrates that the price process $\left\{X_{k}\right\}$ must converge to a Walrasian equilibrium price vector of the underlying economy as long as the weight $\alpha$ satisfies the equality $\alpha=\frac{\lambda}{1+\lambda}$. Beyond the existence of Walrasian equilibrium, this convergence result does not depend on privately known demands and supplies. Instead it depends on the two parameters $\alpha$ and $\lambda$ related to the auction form. If the weight $\alpha$ does not satisfy the equality $\alpha=\frac{\lambda}{1+\lambda}$, then the price process $\left\{X_{k}\right\}$ still converges to a price but it may be higher or lower than the equilibrium price(s). A higher than equilibrium price (i.e. bubble) is obtained when $\alpha<\frac{\lambda}{1+\lambda}$ and a lower than equilibrium price (i.e. crash) is

1 The converse of this claim is not true. See Example 4.9. 
obtained when $\alpha>\frac{\lambda}{1+\lambda}$ by the double auction. For example, $\alpha$ must be right at $\frac{1}{2}$ for $\lambda=1$ in order for the auction to arrive at a Walrasian equilibrium.

For our randomized double auction, our second major result Theorem 4.7 shows that the above result still holds when $\lambda$ is defined by $\lambda=\frac{m}{n} \lim _{k \rightarrow \infty} \frac{b_{k}}{a_{k}}$, where $n$ is the number of buyers (or agents) and $m$ is the number of sellers (or objects), under Assumption 3.3. For example, if $\lim _{k \rightarrow \infty} \frac{b_{k}}{a_{k}}=1$ and $m=2 n$, then $\alpha$ must be right at $\frac{2}{3}$ for the auction to arrive at a Walrasian equilibrium. Once again, this conclusion does not depend on $f_{i}$ and $g_{j}$, which are unknown to the mechanism designer.

The above two results are proved for a general case where there are multiple heterogeneous objects, with each object having a finite number of identical copies. In the general case, $\left\{X_{k}\right\}$ is a sequence of price vectors rather than a sequence of prices. The condition on $\lambda$ is also stated for the case where the limit $\lim _{k \rightarrow \infty} \frac{b_{k}}{a_{k}}$ may not exist.

Noises are identified as a key factor in the formation of bubbles and crashes (Shleifer, 1999). So it is of interest to see how noises may change our results. To examine this issue, we follow Ram et al. (2009) to introduce stochastic noises into buy and sell orders under the two DA mechanisms. Interestingly, our main results still hold for certain noises. This means that not all noises can affect the informational efficiency of a DA mechanism. The relationship between $\alpha$ and $\lambda$ is still the key for the convergence of the price processes under the two DA mechanisms with stochastic noises.

Literature. The benchmark model is the dual problem of the linear programming relaxation in Bikhchandani \& Mamer (1997) that can be reformulated as a convex optimization problem in the price space without constraints by $\mathscr{P}$. An optimal solution to this dual $\mathscr{P}$ (i.e., a minimizer) is a Walrasian equilibrium price vector of the original economy if the zero duality gap condition holds. ${ }^{2}$ They also mention that the ascending price auction designed in Kelso \& Crawford (1982) for a noted many-to-one job matching market can be used to achieve a Walrasian equilibrium price vector for their economy under the gross substitutes condition. A salient feature of the English auction in Kelso \& Crawford (1982) is the constant increase (i.e. step size) of prices for those workers in excess demand at each iteration. Milgrom (2000) studies

2 This dual approach and its related gradient method in search for an equilibrium in games can be traced back to Arrow \& Hurwicz (1957). Beyond its applications in economics and game theory, the dual approach has many applications in other areas (see, e.g., Bertsekas, 2009; Nedić \& Ozdaglar, 2009). 
an economy similar to that in Bikhchandani \& Mamer (1997) and provides an English auction that uses a larger constant price increment in the beginning and a smaller constant price increment near the end for an object in excess demand; the price process generated by such an auction can approach a Walrasian equilibrium at a faster speed when the auction begins, with approximation errors caused by the discrete price increment being reduced near the end of the auction. Xu et al. (2015) provide a subsequent analysis of the two double auctions presented in this paper with constant step sizes and obtain several approximation error bounds, under a more general information communication structure than Figure 1, when $\alpha$ is at random with unknown distributions.

It is often a challenging task to design an efficient auction when the gross substitutes condition is not satisfied and agents have private reservation values over bundles. Sun \& Yang (2009, 2014) develop new auction mechanisms that can approach an efficient allocation when goods are substitutes and complements. There are several other auction mechanisms in the literature, see, e.g., Ausubel (2004), Gul \& Stacchetti (2000), and references in both Milgrom (2000) and in Sun \& Yang (2014).

An English auction is an ascending format of auction in which the price of an item is gradually increased and adjusted in each round according to reported total demand for the given total supply. When the scale of the market is small, this may not cause a problem. However, when it is large, it can be difficult to know the total demand at each round or iteration. A double auction is different, since the process involves pairs of buyers and sellers at each moment in time. A question arises as to whether such an auction process can always achieve a Walrasian equilibrium outcome as is the case with an English auction. The question is a challenge because an equilibrium must be defined with respect to the true demand and supply in totality. But, there is no way to know the total demand and supply in a double auction at any moment in time.

Our double auctions are largely motivated by incremental subgradient methods such as those studied in Kibardin (1980), Nedić \& Bertsekas (2001), Ram et al. (2009), and Solodov \& Zavriev (1998). In an incremental subgradient method, a single sequence of step sizes is used. They are effective in a unilateral market where one single agent updates her information into the price process. However, they are not as useful in a bilateral market situation using double auctions, where there are two sequences of step sizes, one for the buyers and the other for the sellers. A coordination (or "steering") condition between the two step sizes, such as the one on $\lambda$, is required for the convergence of 
the price process. If the $\lambda$ condition fails to hold, our convergence results also fail, as shown in Xu et al. $(2015,2016)$ using numerical simulations. Following the current study, Xu et al. $(2014,2015,2016)$ also demonstrate several convergence results for the two double auctions when agents form some Markovian chains in the iteration process. Moreover, Xu et al. (2015) study the convergence of price processes under the two double auctions when the weight $\alpha$ and two step sizes are independently drawn at random, with unknown distributions.

A major issue with double auctions is proving the existence of a Nash equilibrium; see, e.g., Satterthwaite \& Williams (1989) and Jackson \& Swinkels (2005). However, interdependent reservation values over bundles, assuming unit demand or supply, ${ }^{3}$ are not a major concern for a Nash equilibrium. Our two dynamic double auctions are designed for an environment in which such values play an important role, in a model such as the many-to-one job matching in Kelso \& Crawford (1982) and the multiple unit demand economy in Bikhchandani \& Mamer (1997). These models may also be used for solving resource allocation problems in large scale distributional networks where agents hold dispersed private information; see Subsection 2.2 and e.g., Kelly et al. (1998), Nedić \& Ozdaglar (2009), Ram et al. (2009). This analysis has the potential to provide solutions to real world problems such as the business to business trading in a marketplace or in multiagent coordination systems in artificial intelligence (Xia et al., 2005).

The rest of the paper is organized as follows. Section 2 introduces the model. Section 3 presents the two DA mechanisms and the main assumptions. Section 4 discusses the main results with DA mechanisms without stochastic noises. Section 5 establishes the main results with stochastic noises. Section 6 concludes.

\section{MODEL}

We consider the following general problem (see also Bertsekas, 2012):

$$
\begin{gathered}
\mathscr{P} \quad \operatorname{minimize} F(y) \equiv f(y)+g(y) \\
\text { subject to } y \in Y,
\end{gathered}
$$

3 Kojima \& Yamashita (2016) offer a latest noted exception along the line of Myerson \& Satterthwaite (1983). 
where

$$
f=\sum_{i=1}^{m} f_{i} \text { and } g=\sum_{j=1}^{n} g_{j} .
$$

For all $i=1,2, \cdots, m$ and $j=1,2, \cdots, n, f_{i}: R^{d} \rightarrow R$ and $g_{j}: R^{d} \rightarrow R$ are convex functions and $Y$ is a nonempty convex subset of $R_{+}^{d}$. As Bertsekas (2012) has demonstrated, such a form covers a large class of problems in the literature: a). least squares and related inference problems; b). dual optimization in separable problem; c). problems with many constraints; d). minimization of an expected value - stochastic programming; e). Weber problem in location theory; f). distributed incremental optimization-sensor networks. Here we focus on an application of the problem $\mathscr{P}$ to exchange economies with indivisible assets or goods. Thus we may assume that the price space $Y$ is compact. Since every convex function $\varphi$ on a compact set $Y$ is regular Lipschitzian, the set of subgradients $\partial \varphi(y)$ for every $y \in Y$ is a nonempty, compact, and convex set, where $\partial \varphi(y)$ is defined by

$$
\partial \varphi(y)=\{\eta \mid \varphi(y)+\langle\eta, w-y\rangle \leq \varphi(w), \forall w\}
$$

see e.g., Clarke et al. (1988). For any two regular functions $\varphi$ and $\psi$ at $y$, the sum $\varphi+\psi$ is regular at $y$ and

$$
\partial(\varphi+\psi)(y)=\partial \varphi(y)+\partial \psi(y)
$$

We use the following notation

$$
F^{*}=\inf _{y \in Y} F(y), \quad Y^{*}=\left\{y \in Y \mid F(y)=F^{*}\right\}, \quad \operatorname{dist}\left(y, Y^{*}\right)=\inf _{y^{*} \in Y^{*}}\left\|y-y^{*}\right\|
$$

where $\|\cdot\|$ denotes the Euclidean norm.

\subsection{Benchmark Economies with Multiple Indivisible Objects}

We now show how the problem $\mathscr{P}$ naturally captures a large class of economies or markets typified by the many-to-one job matching model of Kelso \& Crawford (1982) and its related economy with indivisible objects in Bikhchandani $\&$ Mamer (1997).

Let $M=\{1,2, \cdots, m\}$ denote the set of objects and $N=\{1,2, \cdots, n\}$ denote the set of agents. An agent $j$ 's reservation value function $u_{j}: 2^{M} \rightarrow R_{+}$is defined over bundles of objects in $M$ such that $u_{j}(\emptyset)=0$. A feasible allocation 
$Z$ is a partition $\left(Z_{0}, Z_{1}, \cdots, Z_{n}\right)$ of all objects in $M$, in which agent $j$ is allocated with the bundle $Z_{j}$ and $Z_{0}$ is the unsold bundle. Let $\bar{Z}$ denote the set of all feasible allocations. A feasible allocation $Z^{*}$ is Pareto optimal or efficient if

$$
V \equiv \sum_{j=1}^{n} u_{j}\left(Z_{j}^{*}\right) \geq \sum_{j=1}^{n} u_{j}\left(Z_{j}\right), \quad \forall Z \in \bar{Z} .
$$

Given a price vetor $p \in R_{+}^{m}$, agent $j$ 's demand $D_{j}(p)$ consists of all bundles of $M$ that maximize his surplus, i.e., $D_{j}(p)=\left\{S \subset M \mid u_{j}(S)-\sum_{i \in S} p_{i} \geq\right.$ $\left.u_{j}(T)-\sum_{t \in T} p_{t}, \forall T \subset M\right\}$. A pair $(Z, p)$ of a feasible allocation $Z \in \bar{Z}$ and a price vector $p \in R_{+}^{m}$ is a Walrasian equilibrium if $p_{z}=0$ for all $z \in Z_{0}$ and $Z_{j} \in D_{j}(p)$ for all $j \in N$. It is well-known that a Walrasian equilibrium allocation is efficient. Even though an efficient allocation always exists, a Walrasian equilibrium may not exist, due to the nature of interdependent reservation values. Objects that are complement often cause the problem. Two goods satisfy the gross substitutes (GS) condition if a good that is in demand and whose price is not raised will still be in demand if the price of the other good arises. The GS condition of Kelso \& Crawford (1982) is a sufficient condition for existence of a Walrasian equilibrium (Bikhchandani \& Mamer, 1997; Gul \& Stacchetti, 1999). Extensive studies of this condition can be found in Fujishige \& Yang (2003), Hatfield \& Kojima (2010), Hatfield \& Milgrom (2005) among others. Economies that include complementary goods have been studied by Sun \& Yang $(2006,2008,2014)$.

Given $p \in R_{+}^{m}$, define $\pi_{j}(p)=u_{j}(S)-\sum_{i \in S} p_{i}$ for $S \in D_{j}(p)$. Note that $\pi_{j}(p)$ is convex. Then the dual of the linear programming relaxation of the integer programming in Bikhchandani and Mamer can be seen as a convex minimization problem without constraints:

$$
V^{\prime \prime}=\min _{p \in R_{+}^{m}} V(p) \equiv \sum_{i=1}^{m} p_{i}+\sum_{j=1}^{n} \pi_{j}(p) .
$$

Then, we have $V(p) \geq V$ for all $p \in R_{+}^{m}$.

Define $e: 2^{M} \rightarrow R^{m}$ by $e_{i}(S)=1$ for $i \in S$ and $e_{i}(S)=0$ otherwise. We can write the demand correspondence $\hat{D}_{j}: Y \rightarrow R^{m}$ by $\hat{D}_{j}(p)=\left\{e(S) \mid S \in D_{j}(p)\right\}$.

Define $g_{j}(p)=\pi_{j}(p)$ for all $j=1,2, \cdots, n$ and $f_{i}: R_{+}^{n} \rightarrow R$ by $f_{i}(p)=p_{i}$ for all $i=1,2, \cdots, m$. Thus we obtain the general form $F(p)=f(p)+g(p)$ subject to $p \in Y \subset R_{+}^{m}$. Note that the supply of an asset is the interval $[0,1]$ at zero price. It follows from the Fenchel duality that $\bar{c} \hat{o} \hat{S}_{i}(p)=\partial f_{i}(p)$ and 
$\partial g_{j}(p)=-\overline{c o} \hat{D}_{j}(p)$ for all $p \in Y$, where $\overline{c o} C$ denotes the closed convex hull of the set $C$ (Ma \& Nie, 2003). A vector $y$ is an optimal solution in $Y^{*}$ if and only if

$$
0 \in \sum_{i=1}^{n} \overline{c o} \hat{S}_{i}(y)-\sum_{j=1}^{m} \overline{c o} \hat{D}_{j}(y) .
$$

Thus, a price vector $p \in Y$ is at an equilibrium only if $0 \in \partial(f+g)(p)$.

Because $\mathscr{P}$ is a dual of the linear programming relaxation of the primal integer programming in Bikhchandani \& Mamer (1997) for finding an efficient allocation, a solution $y$ to $\mathscr{P}$ is a Walrasian equilibrium if and only if the duality gap is zero, i.e., $V(y)=V$ (Bikhchandani \& Mamer, 1997; Ma \& Nie, 2003). Note that the duality gap approaches zero for a large scale economy (as $m$ and $n$ go to infinite) (Bertsekas, 2009).

\subsection{A Congestion Control Problem with Production}

We introduce a data transmission or congestion control problem on a given network with each link a production function (see, e.g., Kelly et al., 1998). Let $\mathscr{N}=\{1,2, \cdots, n\}$ denote the set of sources and $\mathscr{L}=\{1,2, \cdots, L\}$ the set of all undirected links. Each link $l \in \mathscr{L}$ has an increasing and convex cost function $c_{l}:[0, \infty) \rightarrow[0, \infty)$ such that $c_{l}(0)=0$, i.e., it costs a link $l$ the amount $c_{l}(q)$ to produce capacity $q \geq 0$. Let $L(i) \subset \mathscr{L}$ denote the set of links used by source $i \in \mathscr{N}$. The utility function for a source $i$ is defined by $u_{i}:[0, \infty) \rightarrow[0, \infty)$, which is assumed to be increasing and concave. That is, source $i$ gains a utility $u_{i}\left(x_{i}\right)$ when it sends data at a transmission rate $x_{i}$. Let $N(l)=\{i \in \mathscr{N} \mid l \in L(i)\}$ denote the set of sources that use link $l$. Let $p \in R_{+}^{L}$ denote a price vector, i.e., a link charges $p_{l}$ per unit rate (e.g., packets per second) of data transmission (Kelly et al., 1998). Define $e: 2^{L} \rightarrow R^{L}$ by $e_{l}(S)=1$ if $l \in S$ and $e_{l}(S)=0$ otherwise. Define the supply function $S_{l}: R_{+}^{L} \rightarrow[0, \infty)$ by $S_{l}(p)=\left\{q \mid p_{l} q-c_{l}(q) \geq p_{l} z-c_{l}(z), \forall z \geq 0\right\}$ and the demand function $D_{i}: R_{+}^{L} \rightarrow R_{+}^{L}$ by $D_{i}(p)=\left\{e(L(i)) x_{i} \mid u_{i}\left(x_{i}\right)-x_{i} \sum_{l \in L(i)} p_{l} \geq\right.$ $\left.u_{i}(z)-z \sum_{l \in L(i)} p_{l}, \forall z \geq 0\right\}$. A triplet $(p ; x, q) \in R_{+}^{L} \times R_{+}^{n} \times R_{+}^{L}$ is a network equilibrium if a). $e(L(i)) x_{i} \in D_{i}(p)$ for all $\left.i \in \mathscr{N} ; \mathrm{b}\right) . q_{l} \in S_{l}(p)$ for all $l \in \mathscr{L}$; c). $\sum_{i \in N(l)} x_{i} \leq q_{l}$ for all $l \in \mathscr{L}$. Define $f_{l}(p)=p_{l} q-c_{l}(q), q \in S_{l}(p)$ for all $l \in \mathscr{L}$, and $g_{i}(p)=u_{i}\left(x_{i}\right)-x_{i} \sum_{l \in L(i)} p_{l}, e(L(i)) x_{i} \in D_{i}(p)$ for all $i \in \mathscr{N}$. Let $f(p)=\sum_{l \in \mathscr{L}} f_{l}(p)$ and $g(p)=\sum_{i \in \mathscr{N}} g_{i}(p)$. We obtain the problem $\mathscr{P}$, subject to $p \in R_{+}^{L}$. Thus, one can show that $(p ; x, q)$ is an equilibrium iff $p$ is an optimal solution to the problem $\mathscr{P}$. Note that $Y^{*}$ is nonempty. So an equilibrium always 
exists with transmission rates that are divisible. The problem $\mathscr{P}$ formalized this way is in fact the dual problem of a utility maximization problem on networks. See Kelly et al. (1998), and the examples discussed in Nedić \& Ozdaglar (2009) and Ram et al. (2009), where each link is given with a fixed capacity, no production available. A flexible capacity network is often needed in practice. Under some mild assumptions, the first social welfare theorem holds (i.e., the duality gap is zero). Thus, finding an equilibrium under the problem $\mathscr{P}$ is one way to solve the primal utility maximization problem.

\section{TWO DOUBLE AUCTIONS}

We introduce two new dynamic double auctions. One double auction is designed based on the bilateral cyclic structure presented in Figure 1. The other one is based on a random match between the sellers and the buyers.

\subsection{A Cyclic Double Auction (CDA)}

Assume that $m=n$, which holds if the initial endowments are owned by the $n$ agents, who act as both sellers and buyers.

CDA Mechanism: Let $\Phi_{0, k}=X_{k}$. For $i=1,2, \cdots, m$, let

$$
\begin{aligned}
\psi_{i, k} & =\Phi_{i-1, k}-a_{k} \nabla f_{i}\left(\Phi_{i-1, k}\right), \\
\varphi_{i, k} & =\Phi_{i-1, k}-b_{k} \nabla g_{i}\left(\Phi_{i-1, k}\right), \\
\Phi_{i, k} & =P_{Y}\left(\alpha \psi_{i, k}+(1-\alpha) \varphi_{i, k}\right),
\end{aligned}
$$

where $\nabla f_{i}\left(\Phi_{i-1, k}\right) \in \partial f_{i}\left(\Phi_{i-1, k}\right)$ and $\nabla g_{i}\left(\Phi_{i-1, k}\right) \in \partial g_{i}\left(\Phi_{i-1, k}\right)$. $P_{Y}$ is the Euclidean projection onto $Y$ because the weighted average of the bid and ask prices may be out of $Y$ in the process. Let

$$
X_{k+1}=\Phi_{m, k}
$$

Our explanation of this auction is as follows. $X_{k}$ is the initial price vector for time $k$. We want to obtain $X_{k+1}$ with a round of $m$ iterations. To accomplish the task, the $m$ sellers and $m$ buyers form a cyclic seller ring and a cyclic buyer ring in two random orders, i.e., two random permutations of the $m$ agents. Then we rename these agents in the two rings with $i=1,2, \cdots, m$. Based on the initial price vector $\Phi_{0, k}=X_{k}$, seller 1 and buyer 1 are the first pair to submit an ask 
order and a bid order to determine $\Phi_{1, k}$, which is then communicated to the next pair, seller 2 and buyer 2 . This process continues until it reaches the last pair, seller $m$ and buyer $m$, to obtain $\Phi_{m, k}$. The price vector $X_{k+1}$ is set to be $\Phi_{m, k}$ to end the $m$ iterations in the cycle. After obtaining $X_{k+1}$, the $m$ agents are reshuffled and renamed with two new random permutations and the process proceeds with $X_{k+1}$ in the same manner as with $X_{k}$. The auction starts with a given $X_{0} \in Y \subset R_{++}^{d}$, and generates a sequence of price vectors $\left\{X_{k}\right\}, k \geq 0$.

The ask order for seller $i$ at $k$ consists of a vector of ask prices $\psi_{i, k}$ and an ask size $\nabla f_{i}\left(\Phi_{i-1, k}\right)$, where $\nabla f_{i}\left(\Phi_{i-1, k}\right)$ is on the supply curve $\partial f_{i}\left(\Phi_{i-1, k}\right)$. The relationship between ask prices and sizes is given by equation (14), where $a_{k}$ is the ask step size at round $k$, which is the price decrement for one unit of object for sale. The ask prices are lower than $\Phi_{i-1, k}$, the newly updated prices from the previous pair. The more the seller wants to sell an object, the lower the ask prices.

Similarly, the bid order for buyer $i$ at $k$ consists of a vector of bid prices $\varphi_{i, k}$ and a bid size $\nabla g_{i}\left(\Phi_{i-1, k}\right)$, where $\nabla g_{i}\left(\Phi_{i-1, k}\right)$ is on the demand curve $-\partial g_{i}\left(\Phi_{i-1, k}\right)$. The relationship between bid prices and sizes is given by equation (15), where $b_{k}$ is the bid step size at round $k$, which is the price increment for one unit of object to buy. The bid prices are higher than $\Phi_{i-1, k}$, the newly updated prices from the previous pair. The more the buyer wants to buy an object, the higher the bid prices. The prices $\Phi_{i, k}$ are a weighted average of the ask and bid prices, with a weight in $(0,1)$, as in Chatterjee \& Samuelson (1983).

We provide conditions on the weight $\alpha$ and the two step sizes $\left\{a_{k}\right\}$ and $\left\{b_{k}\right\}$ so that the price process $\left\{X_{k}\right\}$ converges to an optimal solution in $Y^{*}$.

It may be useful to compare CDA with the noted cyclic incremental subgradient method (e.g., Nedić \& Bertsekas, 2001).

Incremental Subgradient Method: Assume $m=n$ and $X_{0} \in Y$. Let $\Phi_{0, k}=X_{k}$. For $i=1,2, \cdots, m$, let

$$
\Phi_{i, k}=P_{Y}\left(\Phi_{i-1, k}-a_{k} \nabla\left(f_{i}+g_{i}\right)\left(\Phi_{i-1, k}\right)\right),
$$

where $\nabla\left(f_{i}+g_{i}\right)\left(\Phi_{i-1, k}\right) \in \partial\left(f_{i}+g_{i}\right)\left(\Phi_{i-1, k}\right)$. Let $X_{k+1}=\Phi_{m, k}$. $P_{Y}$ is the Euclidean projection onto the set $Y$.

In the incremental subgradient method, $m$ agents form a single ring in an arbitrary order (Figure 2). Prices are iterated along the ring one agent at a time. At each iteration, only one agent $i$ reveals his buy size in $-\partial g_{i}\left(\Phi_{i-1, k}\right)$ 
Figure 2. An Iteration under the Incremental Subgradient Method.

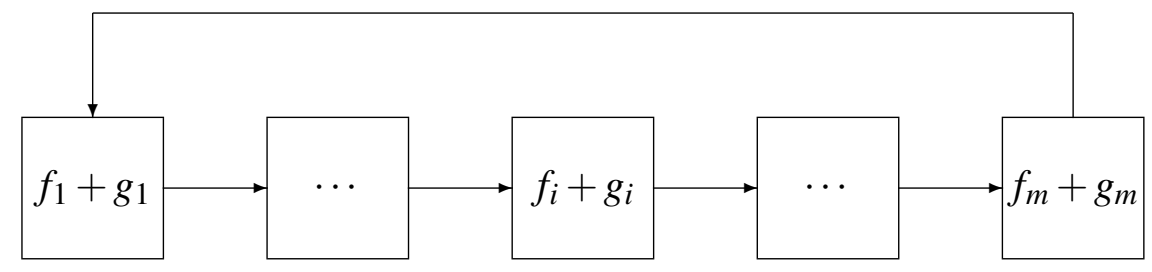

together with his sell size in $\partial f_{i}\left(\Phi_{i-1, k}\right)$ at prices $\Phi_{i-1, k}$. A key feature of their algorithm is that adjustment in prices at each iteration depends on the chosen step size $a_{k}$ and the individual excess supply $\nabla\left(f_{i}+g_{i}\right)\left(\Phi_{i-1, k}\right)$. They show that if the step size $\left\{a_{k}\right\}$ is diminishing, their algorithm generates a sequence of prices that converges to an equilibrium in $Y^{*}$. CDA is different from the incremental subgradient method in two ways. First, the buyer and seller cyclic rings are different. We consider $f$ and $g$ as two different sides of the market. Second, there are two sequences of step sizes $\left\{a_{k}\right\}$ and $\left\{b_{k}\right\}$ in CDA. This makes the convergence results for the incremental subgradient method inapplicable to CDA because there is a new $\lambda$ condition. Even if the $\lambda$ condition is satisfied, the weight $\alpha$ and the parameter $\lambda$ must be in a right combination so that the process $\left\{X_{k}\right\}$ can converge to an optimal solution in $Y^{*}$. We need to consider two step sizes because the market using double auctions is bilateral, in contrast to the unilateral market under the incremental subgradient method.

\subsection{A Randomized Double Auction (RDA)}

Let $w_{k}$ be a random variable taking equiprobable values from the set $\{1,2, \cdots, m\}$ and $w_{k}^{\prime}$ be a random variable taking equiprobable values from the set $\{1,2, \cdots, n\}$. Let $\nabla f_{w_{k}}\left(X_{k}\right) \in \partial f_{w_{k}}\left(X_{k}\right)$ and $\nabla g_{w_{k}^{\prime}}\left(X_{k}\right) \in \partial g_{w_{k}^{\prime}}\left(X_{k}\right)$, where if $w_{k}$ takes a value $i$, then the vector $\partial f_{w_{k}}\left(X_{k}\right)$ is $\partial f_{i}\left(X_{k}\right)$, similarly for $g$. Here we do not need to assume that $m=n$.

Our sequence $\left\{X_{k}\right\}$ is generated by RDA mechanism as below.

RDA Mechanism: Given $X_{k}$, let

$$
\psi_{w_{k}, k+1}=X_{k}-a_{k} \nabla f_{w_{k}}\left(X_{k}\right)
$$




$$
\varphi_{w_{k}^{\prime}, k+1}=X_{k}-b_{k} \nabla g_{w_{k}^{\prime}}\left(X_{k}\right)
$$

Let

$$
X_{k+1}=P_{Y}\left(\alpha \psi_{w_{k}, k+1}+(1-\alpha) \varphi_{w_{k}^{\prime}, k+1}\right),
$$

where $\alpha \in(0,1)$ and $P_{Y}$ is the Euclidean projection onto $Y$.

Our explanation of this auction is as follows. $X_{k}$ is the price vector at time $k$. We want to obtain $X_{k+1}$ by incorporating individual demand and supply information from a pair of buyer and seller, with the seller randomly selected from the set of sellers and the buyer randomly selected from the set of buyers. The bid prices and bid size as well as the ask price and ask bid are determined in the same way as in CDA. This is equivalent to the case where a seller randomly matches with a buyer. Again, the auction starts with $X_{0} \in Y$. Then (9)-(11) generate a sequence of prices $X_{k}, k=1,2, \cdots$.

We are interested in the convergence of $\left\{X_{k}\right\}$ and the conditions under which $\left\{X_{k}\right\}$ converges to an optimal solution in $Y^{*}$. Because $f$ and $g$ are privately known to agents, not to the mechanism designer, our conditions cannot be imposed on $f$ and $g$. This provides a challenge because information about $f$ and $g$ has never been revealed in totality in the two auction processes. The two auction forms are in spirit close to the decentralized market mechanism as stated in A. Smith (1776) and Hayek (1945), in contrast to a centralized market mechanism.

\subsection{Key Assumptions}

We assume that the step sizes satisfy the following diminishing conditions in Assumption 3.1, which is standard for many convergence results in the literature. Assumptions 3.2 and 3.3 are the two new assumptions for two double auctions CDA and RDA. To achieve an equilibrium in $Y^{*}$, we must have a right combination of $\alpha$ and $\lambda$.

Assumption 3.1 (Diminishing step sizes). Assume that the two sequences $\left\{a_{k}\right\}$ and $\left\{b_{k}\right\}$ of step sizes are such that (i). $a_{k}>0$ and $b_{k}>0$; (ii). $\sum_{k=0}^{\infty} a_{k}=+\infty$ and $\sum_{k=0}^{\infty} b_{k}=+\infty$; (iii). $\sum_{k=0}^{\infty} a_{k}^{2}<+\infty$ and $\sum_{k=0}^{\infty} b_{k}^{2}<+\infty$.

Assumption 3.2. Assume that the two sequences $\left\{a_{k}\right\}$ and $\left\{b_{k}\right\}$ of step sizes 
are such that there exists some positive $\lambda$ to ensure

$$
\sum_{k=0}^{\infty}\left|b_{k}-\lambda a_{k}\right|<+\infty .
$$

Assumption 3.3. Assume that the two sequences $\left\{a_{k}\right\}$ and $\left\{b_{k}\right\}$ of step-sizes are such that there exists some positive $\lambda$ to ensure

$$
\sum_{k=0}^{\infty}\left|\frac{b_{k}}{n}-\lambda \frac{a_{k}}{m}\right|<+\infty .
$$

Note the difference between Assumptions 3.2 and 3.3. Assumption 3.2 is for CDA where $m=n$, while Assumption 3.3 is for RDA where $m$ may be different from $n$. These $\lambda$ conditions (12) and (13) will be discussed in Subsection 4.4.

\section{MAIN RESULTS}

In this section we prove our two main results for CDA (5)-(8) and RDA (9)-(11). Lemma 4.1 below is the key for both mechanisms.

\subsection{Main Result: CDA}

Since $Y$ is compact, there exist scalars $C_{1}, C_{2}, \cdots, C_{m}$ and $D_{1}, D_{2}, \cdots, D_{m}$ such that

$$
\|h\| \leq C_{i}, \quad \forall h \in \partial f_{i}\left(X_{k}\right) \cup \partial f_{i}\left(\Phi_{i-1, k}\right), i=1,2, \cdots, m, k=0,1,2, \cdots
$$

and

$$
\|\ell\| \leq D_{i}, \quad \forall \ell \in \partial g_{i}\left(X_{k}\right) \cup \partial g_{i}\left(\Phi_{i-1, k}\right), i=1,2, \cdots, m, k=0,1,2, \cdots .
$$

Note that $m=n$.

Lemma 4.1. Let $\left\{X_{k}\right\}$ be the sequence generated by CDA (5)-(8). Then for all $y \in Y$ and $k \geq 0$, we have

$$
\begin{aligned}
\left\|X_{k+1}-y\right\|^{2} & \leq\left\|X_{k}-y\right\|^{2}-2 a_{k} \alpha\left(f\left(X_{k}\right)-f(y)\right)-2 b_{k}(1-\alpha)\left(g\left(X_{k}\right)-g(y)\right) \\
& +\left(\alpha a_{k} C+(1-\alpha) b_{k} D\right)^{2}
\end{aligned}
$$


where $C=\sum_{i=1}^{m} C_{i}$ and $D=\sum_{i=1}^{m} D_{i}$.

Remark. This lemma shows that the quadratic distance of the price process $\left\{X_{k}\right\}$ to an equilibrium in $Y^{*}$ can be bounded and that it is possible for the price process to approach an equilibrium in $Y^{*}$. Nonetheless, a naive choice $y$ in $Y^{*}$ does not work because of this term $\left[a_{k} \alpha f\left(X_{k}\right)+b_{k}(1-\right.$ $\left.\alpha) g\left(X_{k}\right)\right]-\left[a_{k} \alpha f(y)+b_{k}(1-\alpha) g(y)\right]$, which is not a sum form $f+g$ as defined in $\mathscr{P}$. For a choice $y$ in $Y^{*}$, there is no guarantee that the term $\left[a_{k} \alpha f\left(X_{k}\right)+b_{k}(1-\alpha) g\left(X_{k}\right)\right]-\left[a_{k} \alpha f(y)+b_{k}(1-\alpha) g(y)\right]$ is always nonnegative. This is why the convergence results for the incremental subgradient method with diminishing step sizes in Nedić \& Bertsekas (2001) does not apply to CDA.

Proof. Denote $h_{i, k}=\nabla f_{i}\left(\Phi_{i-1, k}\right)$ and $\ell_{i, k}=\nabla g_{i}\left(\Phi_{i-1, k}\right)$ for all $i=1,2, \cdots, m$. By the non-expansive property of projection, we have

$$
\begin{aligned}
\left\|\Phi_{i, k}-y\right\|^{2} \leq & \left\|\alpha \psi_{i, k}+(1-\alpha) \varphi_{i, k}-y\right\|^{2} \\
= & \left\|\alpha\left(\psi_{i, k}-y\right)+(1-\alpha)\left(\varphi_{i, k}-y\right)\right\|^{2} \\
= & \alpha^{2}\left\|\psi_{i, k}-y\right\|^{2}+(1-\alpha)^{2}\left\|\varphi_{i, k}-y\right\|^{2} \\
& +2 \alpha(1-\alpha)\left\langle\left(\psi_{i, k}-y\right),\left(\varphi_{i, k}-y\right)\right\rangle \\
= & \alpha^{2}\left\|\Phi_{i-1, k}-y-a_{k} h_{i, k}\right\|^{2}+(1-\alpha)^{2}\left\|\Phi_{i-1, k}-y-b_{k} \ell_{i, k}\right\|^{2} \\
& +2 \alpha(1-\alpha)\left\langle\left(\Phi_{i-1, k}-y-a_{k} h_{i, k}\right),\left(\Phi_{i-1, k}-y-b_{k} \ell_{i, k}\right)\right\rangle \\
= & \alpha^{2}\left\|\Phi_{i-1, k}-y\right\|^{2}-2 a_{k} \alpha^{2}\left\langle h_{i, k},\left(\Phi_{i-1, k}-y\right)\right\rangle+\alpha^{2} a_{k}^{2}\left\|h_{i, k}\right\|^{2} \\
& +(1-\alpha)^{2}\left\|\Phi_{i-1, k}-y\right\|^{2}-2 b_{k}(1-\alpha)^{2}\left\langle\ell_{i, k},\left(\Phi_{i-1, k}-y\right)\right\rangle \\
& +(1-\alpha)^{2} b_{k}^{2}\left\|\ell_{i, k}\right\|^{2}+2 \alpha(1-\alpha)\left\|\Phi_{i-1, k}-y\right\|^{2} \\
& -2 \alpha(1-\alpha) a_{k}\left\langle h_{i, k},\left(\Phi_{i-1, k}-y\right)\right\rangle \\
& -2 \alpha(1-\alpha) b_{k}\left\langle\ell_{i, k},\left(\Phi_{i-1, k}-y\right)\right\rangle \\
& +2 \alpha(1-\alpha) a_{k} b_{k}\left\langle h_{i, k}, \ell_{i, k}\right\rangle \\
= & \left\|\Phi_{i-1, k}-y\right\|^{2}-2(1-\alpha) b_{k}\left\langle\ell_{i, k},\left(\Phi_{i-1, k}-y\right)\right\rangle \\
& +\left\|\alpha a_{k} h_{i, k}+(1-\alpha) b_{k} \ell_{i, k}\right\|^{2}-2 \alpha a_{k}\left\langle h_{i, k},\left(\Phi_{i-1, k}-y\right)\right\rangle
\end{aligned}
$$

Since we have $\left\|h_{i, k}\right\| \leq C_{i},\left\|\ell_{i, k}\right\| \leq D_{i}$ for all $k=0,1,2, \cdots$, we obtain

$$
\begin{aligned}
\left\|\Phi_{i, k}-y\right\|^{2} \leq & \left\|\Phi_{i-1, k}-y\right\|^{2}+\left(\alpha a_{k} C_{i}+(1-\alpha) b_{k} D_{i}\right)^{2} \\
& -2\left\langle\left(\alpha a_{k} h_{i, k}+(1-\alpha) b_{k} \ell_{i, k}\right),\left(\Phi_{i-1, k}-y\right)\right\rangle
\end{aligned}
$$


Summing over $i=1,2, \cdots, m$, we get

$$
\begin{aligned}
\sum_{i=1}^{m}\left\|\Phi_{i, k}-y\right\|^{2} \leq & \sum_{i=1}^{m}\left\|\Phi_{i-1, k}-y\right\|^{2}+\sum_{i=1}^{m}\left(\alpha a_{k} C_{i}+(1-\alpha) b_{k} D_{i}\right)^{2} \\
& -2 \sum_{i=1}^{m}\left\langle\left(\alpha a_{k} h_{i, k}+(1-\alpha) b_{k} \ell_{i, k}\right),\left(\Phi_{i-1, k}-y\right)\right\rangle
\end{aligned}
$$

So we have

$$
\begin{aligned}
\left\|X_{k+1}-y\right\|^{2} \leq & \left\|X_{k}-y\right\|^{2}+\sum_{i=1}^{m}\left(\alpha a_{k} C_{i}+(1-\alpha) b_{k} D_{i}\right)^{2} \\
& -2 \sum_{i=1}^{m}\left\langle\left(\alpha a_{k} h_{i, k}+(1-\alpha) b_{k} \ell_{i, k}\right),\left(\Phi_{i-1, k}-y\right)\right\rangle
\end{aligned}
$$

By the definition of subgradients $h_{i, k}$ and $\ell_{i, k}$,

$$
\left\langle h_{i, k},\left(y-\Phi_{i-1, k}\right)\right\rangle \leq f_{i}(y)-f_{i}\left(\Phi_{i-1, k}\right)
$$

and

$$
\left\langle\ell_{i, k},\left(y-\Phi_{i-1, k}\right)\right\rangle \leq g_{i}(y)-g_{i}\left(\Phi_{i-1, k}\right) .
$$

Then

$$
\begin{aligned}
\left\|X_{k+1}-y\right\|^{2} \leq & \left\|X_{k}-y\right\|^{2}-2(1-\alpha) b_{k} \sum_{i=1}^{m}\left(g_{i}\left(\Phi_{i-1, k}\right)-g_{i}(y)\right) \\
& +\sum_{i=1}^{m}\left(\alpha a_{k} C_{i}+(1-\alpha) b_{k} D_{i}\right)^{2}-2 \alpha a_{k} \sum_{i=1}^{m}\left(f_{i}\left(\Phi_{i-1, k}\right)-f_{i}(y)\right)
\end{aligned}
$$

So

$$
\begin{aligned}
\left\|X_{k+1}-y\right\|^{2} \leq & \left\|X_{k}-y\right\|^{2}-2 \alpha a_{k}\left(f\left(X_{k}\right)-f(y)\right)-2(1-\alpha) b_{k}\left(g\left(X_{k}\right)-g(y)\right) \\
& -2 \alpha a_{k} \sum_{i=1}^{m}\left(f_{i}\left(\Phi_{i-1, k}\right)-f_{i}\left(X_{k}\right)\right)+\sum_{i=1}^{m}\left(\alpha a_{k} C_{i}+(1-\alpha) b_{k} D_{i}\right)^{2} \\
& -2(1-\alpha) b_{k} \sum_{i=1}^{m}\left(g_{i}\left(\Phi_{i-1, k}\right)-g_{i}\left(X_{k}\right)\right)
\end{aligned}
$$

Next we need to estimate $f_{i}\left(\Phi_{i-1, k}\right)-f_{i}\left(X_{k}\right)$ and $g_{i}\left(\Phi_{i-1, k}\right)-g_{i}\left(X_{k}\right)$.

Lemma 4.1.1. $\left\|\Phi_{i-1, k}-X_{k}\right\| \leq \sum_{j=1}^{i-1}\left(\alpha a_{k} C_{j}+(1-\alpha) b_{k} D_{j}\right)$. 
Proof. We show Lemma 4.1.1 by induction. Note that $\Phi_{0, k}-X_{k}=0$. Assume that it holds for $i-1$. Then

$$
\begin{aligned}
\left\|\Phi_{i, k}-X_{k}\right\| & =\left\|\left(\alpha \psi_{i, k}+(1-\alpha) \varphi_{i, k}\right)-X_{k}\right\| \\
& \leq \alpha\left\|\psi_{i, k}-X_{k}\right\|+(1-\alpha)\left\|\varphi_{i, k}-X_{k}\right\| \\
& \leq \alpha\left\|\Phi_{i-1, k}-a_{k} h_{i, k}-X_{k}\right\|+(1-\alpha)\left\|\Phi_{i-1, k}-b_{k} \ell_{i, k}-X_{k}\right\| \\
& \leq\left\|\Phi_{i-1, k}-X_{k}\right\|+\alpha a_{k} C_{i}+(1-\alpha) b_{k} D_{i} \text { by induction hypothesis } \\
& \leq \sum_{j=1}^{i-1}\left(\alpha a_{k} C_{j}+(1-\alpha) b_{k} D_{j}\right)+\alpha a_{k} C_{i}+(1-\alpha) b_{k} D_{i} .
\end{aligned}
$$

This completes the proof of Lemma 4.1.1.

So

$$
\left\|f_{i}\left(\Phi_{i-1, k}\right)-f_{i}\left(X_{k}\right)\right\| \leq \sum_{j=1}^{i-1} C_{i}\left(\alpha a_{k} C_{j}+(1-\alpha) b_{k} D_{j}\right)
$$

and

$$
\left\|g_{i}\left(\Phi_{i-1, k}\right)-g_{i}\left(X_{k}\right)\right\| \leq \sum_{j=1}^{i-1} D_{i}\left(\alpha a_{k} C_{j}+(1-\alpha) b_{k} D_{j}\right) .
$$

Plugging into (14), we have

$$
\begin{aligned}
\left\|X_{k+1}-y\right\|^{2} \leq & \left\|X_{k}-y\right\|^{2}-2 \alpha a_{k}\left(f\left(X_{k}\right)-f(y)\right)-2(1-\alpha) b_{k}\left(g\left(X_{k}\right)-g(y)\right) \\
& +2 \sum_{i=1}^{m}\left(\alpha a_{k} C_{i}+(1-\alpha) b_{k} D_{i}\right) \sum_{j=1}^{i-1}\left(\alpha a_{k} C_{j}+(1-\alpha) b_{k} D_{j}\right) \\
& +\sum_{i=1}^{m}\left(\alpha a_{k} C_{i}+(1-\alpha) b_{k} D_{i}\right)^{2} \\
= & \left\|X_{k}-y\right\|^{2}-2 \alpha a_{k}\left(f\left(X_{k}\right)-f(y)\right)-2(1-\alpha) b_{k}\left(g\left(X_{k}\right)-g(y)\right) \\
& +\left(\sum_{i=1}^{m}\left(\alpha a_{k} C_{i}+(1-\alpha) b_{k} D_{i}\right)\right)^{2} \\
= & \left\|X_{k}-y\right\|^{2}-2 \alpha a_{k}\left(f\left(X_{k}\right)-f(y)\right)-2(1-\alpha) b_{k}\left(g\left(X_{k}\right)-g(y)\right) \\
& +\left(\alpha a_{k} C+(1-\alpha) b_{k} D\right)^{2} .
\end{aligned}
$$

This completes the proof of Lemma 4.1.

Remark. To apply Lemma 4.1, we must choose a right $y$ for CDA. To do so, we need to define the following $\mathscr{P}(\alpha, \lambda)$ :

$$
\mathscr{P}(\alpha, \lambda) \quad \operatorname{minimize}_{y \in Y} F(y, \alpha, \lambda) \equiv(\alpha f+\lambda(1-\alpha) g)(y)
$$


where $\alpha \in(0,1)$. The parameter $\lambda$ is some positive scalar. Then we introduce the following notation

$$
F^{*}(\alpha, \lambda)=\inf _{y \in Y} F(y, \alpha, \lambda), \quad Y^{*}(\alpha, \lambda)=\left\{y \in Y \mid F(y, \alpha, \lambda)=F^{*}(\alpha, \lambda)\right\}
$$

and $\operatorname{dist}\left(x, Y^{*}(\alpha, \lambda)\right)$, the Euclidean distance. Note that $Y^{*}$ and $Y^{*}(\alpha, \lambda)$ are related but they are not the same, depending on $\alpha$ and $\lambda$.

Proposition 4.2. Let Assumptions 3.1 and 3.2 hold. Let $\left\{X_{k}\right\}$ be the price sequence generated by CDA (5)-(8). Then

$$
\liminf _{k \rightarrow \infty} \operatorname{dist}\left(X_{k}, Y^{*}(\alpha, \lambda)\right)=0 .
$$

Proof. From Lemma 4.1, we obtain for all $y^{*} \in Y^{*}(\alpha, \lambda)$ and $k \geq 0$,

$$
\begin{aligned}
\left\|X_{k+1}-y^{*}\right\|^{2} \leq & \left\|X_{k}-y^{*}\right\|^{2}-2\left(b_{k}-\lambda a_{k}\right)(1-\alpha)\left(g\left(X_{k}\right)-g\left(y^{*}\right)\right) \\
& -2 a_{k}\left[(\alpha f+(1-\alpha) \lambda g)\left(X_{k}\right)-(\alpha f+(1-\alpha) \lambda g)\left(y^{*}\right)\right] \\
& +\left(\alpha a_{k} C+(1-\alpha) b_{k} D\right)^{2} .
\end{aligned}
$$

Since $Y$ is compact, $g$ is continuous, image $(g(Y))$ is bounded. That means there exists $M>0$ such that $|g(y)| \leq M$ for all $y \in Y$. Hence, for any $N=$ $1,2, \cdots$,

$$
\begin{aligned}
0 \leq & \left\|X_{N+1}-y^{*}\right\|^{2} \leq\left\|X_{0}-y^{*}\right\|^{2}+\sum_{k=0}^{N}\left(\alpha a_{k} C+(1-\alpha) b_{k} D\right)^{2} \\
& -2 \sum_{k=0}^{N} a_{k}\left[(\alpha f+(1-\alpha) \lambda g)\left(X_{k}\right)-(\alpha f+(1-\alpha) \lambda g)\left(y^{*}\right)\right] \\
& +2\left(\sum_{k=0}^{N}\left|b_{k}-\lambda a_{k}\right|\right) \cdot(1-\alpha) \cdot 2 M \\
= & I-I I+I I I+I V .
\end{aligned}
$$

I is a constant. When $N$ goes to infinity, $I I I<+\infty$ since $\sum_{k=0}^{\infty}\left|b_{k}-\lambda a_{k}\right|<+\infty$ and

$$
I V \leq 2\left(\alpha^{2} C^{2} \sum_{k=0}^{\infty} a_{k}^{2}+(1-\alpha)^{2} D^{2} \sum_{k=0}^{\infty} b_{k}^{2}\right)<+\infty .
$$


Thus, $I I<+\infty$. We obtain

$$
\liminf _{k \rightarrow \infty}\left[(\alpha f+(1-\alpha) \lambda g)\left(X_{k}\right)-(\alpha f+(1-\alpha) \lambda g)\left(y^{*}\right)\right]=0
$$

Otherwise, $\exists \delta>0$ such that for any natural number $k$

$$
(\alpha f+(1-\alpha) \lambda g)\left(X_{k}\right)-(\alpha f+(1-\alpha) \lambda g)\left(y^{*}\right)>\delta
$$

And then $I I>\delta \sum_{k=0}^{\infty} a_{k}=+\infty$, a contradiction.

Now take a subsequence $\left\{X_{n_{k}}\right\}$ of $\left\{X_{k}\right\}$ such that

$$
0 \leq(\alpha f+(1-\alpha) \lambda g)\left(X_{n_{k}}\right)-(\alpha f+(1-\alpha) \lambda g)\left(y^{*}\right)<\frac{1}{k} .
$$

By the fact that $Y$ is compact, $\left\{X_{n_{k}}\right\}$ has at least one accumulation point $y_{0}$, say, and since $\alpha f+(1-\alpha) \lambda g$ is continuous, we have that

$$
\lim _{k \rightarrow \infty}(\alpha f+(1-\alpha) \lambda g)\left(X_{n_{k}}\right)=(\alpha f+(1-\alpha) \lambda g)\left(y_{0}\right) .
$$

By the definition of $\left\{X_{n_{k}}\right\}$, we know that

$$
\lim _{k \rightarrow \infty}(\alpha f+(1-\alpha) \lambda g)\left(X_{n_{k}}\right)=(\alpha f+(1-\alpha) \lambda g)\left(y^{*}\right), y^{*} \in Y^{*}(\alpha, \lambda) .
$$

Hence, $y_{0} \in Y^{*}(\alpha, \lambda)$. So

$$
\liminf _{k \rightarrow \infty} \operatorname{dist}\left(X_{k}, Y^{*}(\alpha, \lambda)\right)=0
$$

This completes the proof.

Proposition 4.3. Let Assumptions 3.1 and 3.2 hold. Then the sequence $\left\{X_{k}\right\}$ in Proposition 4.2 converges to an optimal solution $y_{0} \in Y^{*}(\alpha, \lambda)$.

Proof. Let

$$
\begin{aligned}
\delta_{k}= & 2 a_{k}\left[(\alpha f+(1-\alpha) \lambda g)\left(X_{k}\right)-(\alpha f+(1-\alpha) \lambda g)\left(y_{0}\right)\right] \\
& +2\left(\left|b_{k}-\lambda a_{k}\right|\right) \cdot(1-\alpha) \cdot 2 M+\left(\alpha a_{k} C+(1-\alpha) b_{k} D\right)^{2}>0 .
\end{aligned}
$$


Then $\sum_{k=0}^{\infty} \delta_{k}=I I+I I I+I V<+\infty$ (see the proof of Proposition 4.2). We also have that

$$
\begin{aligned}
\left\|X_{k+1}-y_{0}\right\|^{2} \leq & \left\|X_{k}-y_{0}\right\|^{2}+\left(\alpha a_{k} C+(1-\alpha) b_{k} D\right)^{2} \\
& -2 a_{k}\left[(\alpha f+(1-\alpha) \lambda g)\left(X_{k}\right)-(\alpha f+(1-\alpha) \lambda g)\left(y_{0}\right)\right] \\
& +2\left(\left|b_{k}-\lambda a_{k}\right|\right) \cdot(1-\alpha) \cdot 2 M \\
\leq & \left\|X_{k}-y_{0}\right\|^{2}+\delta_{k} .
\end{aligned}
$$

Then applying Proposition 1.3 in Correa \& Lemaréchal (1993) to the result in Proposition 4.2, we have that

$$
\lim _{k \rightarrow \infty} X_{k}=y_{0}
$$

Theorem 4.4. Let Assumptions 3.1 and 3.2 hold. Assume that $\alpha=\frac{\lambda}{1+\lambda}$ and $\alpha \in(0,1)$. Then the sequence $\left\{X_{k}\right\}$ generated by CDA (5)-(8) converges to an optimal solution in $Y^{*}$.

Proof. It follows from Proposition 4.3 and the definition of $Y^{*}(\alpha, \lambda)$, which is the same as $Y^{*}$ when $(1-\alpha) \lambda=\alpha$ holds. This completes the proof.

Remark. Suppose the $\operatorname{limit}_{k \rightarrow \infty} \frac{b_{k}}{a_{k}}$ exists and Assumptions 3.1 and 3.2 hold. Then we will see in Section 4.4 that $\lambda=\lim _{k \rightarrow \infty} \frac{b_{k}}{a_{k}}$. Assume that $\lambda=1$. Then Theorem 4.4 shows that the price process $\left\{X_{k}\right\}$ converges to a Walrasian equilibrium of the underlying economy if $\alpha=\frac{1}{2}$. Once $\lambda$ changes, we must also change $\alpha$ accordingly in order to achieve a Walrasian equilibrium. Otherwise the price process $\left\{X_{k}\right\}$ still converges but it may not converge to a Walrasian equilibrium of the original economy. In particular, Theorem 4.4 fails if Assumption 3.2 does not hold, as shown in Xu et al. $(2015,2016)$ by numerical simulations.

\subsection{Main Result for RDA}

Assumption 4.5. The sequence $\left\{w_{k}\right\}\left(\left\{w_{k}^{\prime}\right\}\right)$ is a sequence of independent random variables, each uniformly distributed over the set $\{1,2, \cdots, m\}(\{1,2, \cdots, n\})$. 
Furthermore, the two sequences $\left\{w_{k}\right\}$ and $\left\{w_{k}^{\prime}\right\}$ are independent of the sequence $\left\{X_{k}\right\}$.

Since $Y$ is compact, $f$ and $g$ are regular, we have that the two sets of subgradients $\left\{\nabla f_{w_{k}}\left(X_{k}\right), k=0,1,2, \cdots\right\}$ and $\left\{\nabla g_{w_{k}^{\prime}}\left(X_{k}\right), k=0,1,2, \cdots\right\}$ are bounded. That is, there exist some positive constants $C_{0}$ and $D_{0}$ such that, with probability $1,\left\|\nabla f_{w_{k}}\left(X_{k}\right)\right\| \leq C_{0}$ and $\left\|\nabla g_{w_{k}^{\prime}}\left(X_{k}\right)\right\| \leq D_{0}, \forall k \geq 0$.

Proposition 4.6. Let Assumptions 3.1, 3.3, and 4.5 hold. Then the sequence $\left\{X_{k}\right\}$ generated by $R D A(9)-(11)$ converges to an optimal solution in $Y^{*}(\alpha, \lambda)$ with probability 1 .

Proof. Since $Y$ is compact and $g$ is continuous, there exists $M$ such that $|g(y)| \leq M$ for all $y \in Y$. We obtain for all $k$ and $y \in Y^{*}(\alpha, \lambda)$, as in the proof of Proposition 4.2, by applying Lemma 4.1 to the case with $m=1$ :

$$
\begin{aligned}
E\left\{\left\|X_{k+1}-y\right\|^{2} \mid \mathscr{F}_{k}\right\} \leq & \left\|X_{k}-y\right\|^{2}-2(1-\alpha) \frac{b_{k}}{n}\left(g\left(X_{k}\right)-g(y)\right) \\
& +\left(\alpha a_{k} C_{0}+(1-\alpha) b_{k} D_{0}\right)^{2}-2 \alpha \frac{a_{k}}{m}\left(f\left(X_{k}\right)-f(y)\right) \\
\leq & \left\|X_{k}-y\right\|^{2}+\left(\alpha a_{k} C_{0}+(1-\alpha) b_{k} D_{0}\right)^{2} \\
& -\frac{2 a_{k}}{m}\left[(\alpha f+\lambda(1-\alpha) g)\left(X_{k}\right)-(\alpha f+\lambda(1-\alpha) g)(y)\right] \\
& +2\left|\frac{b_{k}}{n}-\lambda \frac{a_{k}}{m}\right| \cdot\left|g\left(X_{k}\right)-g(y)\right| \\
\leq & \left\|X_{k}-y\right\|^{2}+4 M\left|\frac{b_{k}}{n}-\lambda \frac{a_{k}}{m}\right|+\left(\alpha a_{k} C_{0}+(1-\alpha) b_{k} D_{0}\right)^{2} \\
& -\frac{2 a_{k}}{m}\left[(\alpha f+\lambda(1-\alpha) g)\left(X_{k}\right)-(\alpha f+\lambda(1-\alpha) g)(y)\right]
\end{aligned}
$$

where $\mathscr{F}_{k}=\left\{X_{0}, X_{1}, \cdots, X_{k}\right\}$.

Two definitions are in order. A sample path is a sequence of $\left\{X_{k}\right\}$. For each $y^{*} \in Y^{*}(\alpha, \lambda)$, let $\Omega_{y^{*}}$ denote the set containing all sample paths $\left\{X_{k}\right\}$ such that

$$
2 \sum_{k=0}^{\infty} \frac{a_{k}}{m}\left[(\alpha f+\lambda(1-\alpha) g)\left(X_{k}\right)-(\alpha f+\lambda(1-\alpha) g)\left(y^{*}\right)\right] \leq K<+\infty,
$$

and that $\left\{\left\|X_{k}-y^{*}\right\|\right\}$ converges. We need the following. 
Supermartingale Convergence Theorem (Theorem 3.1 in Nedić \& Bertsekas, 2001). Let $X_{k}, Z_{k}$ and $W_{k}, k=0,1,2, \cdots$, be three sequences of random variables and let $\mathscr{F}_{k}, k=0,1,2, \cdots$, be sets of random variables such that $\mathscr{F}_{k} \subset \mathscr{F}_{k+1}$ for all $k$. Suppose that:

(a) The random variables $X_{k}, Z_{k}$, and $W_{k}$ are nonnegative, and are functions of the random variables in $\mathscr{F}_{k}$.

(b) For each $k$, we have $E\left\{X_{k+1} \mid \mathscr{F}_{k}\right\} \leq X_{k}-Z_{k}+W_{k}$.

(c) There holds $\sum_{k=0}^{\infty} W_{k}<\infty$.

Then, we have $\sum_{k=0}^{\infty} Z_{k}<\infty$, and the sequence $X_{k}$ converges to a nonnegative random variable $X$, with probability 1 .

By the supermartingale convergence theorem, for each $y^{*} \in Y^{*}(\alpha, \lambda)$, we have that $\Omega_{y^{*}}$ is a set of probability 1. Let $\left\{v_{i}\right\}$ be a countable subset of the relative interior $\operatorname{relint}\left(Y^{*}(\alpha, \lambda)\right)$ that is dense in $Y^{*}(\alpha, \lambda)$. Define $\Omega=\bigcap_{i=1}^{\infty} \Omega_{v_{i}}$. Then $\Omega$ has probability 1 since

$$
\operatorname{Prob}\left(\bigcup_{i}^{\infty} \bar{\Omega}_{v_{i}}\right) \leq \sum_{i=1}^{\infty} \operatorname{Prob}\left(\bar{\Omega}_{v_{i}}\right)=0 .
$$

For each sample path in $\Omega$, the sequence $\left\|X_{k}-v_{i}\right\|$ converges so that $\left\{X_{k}\right\}$ is bounded. By

$$
2 \sum_{k=0}^{\infty} \frac{a_{k}}{m}\left[(\alpha f+\lambda(1-\alpha) g)\left(X_{k}\right)-(\alpha f+\lambda(1-\alpha) g)(y)\right] \leq K<+\infty,
$$

we have

$$
\lim _{k \rightarrow \infty}\left[(\alpha f+\lambda(1-\alpha) g)\left(X_{k}\right)-(\alpha f+\lambda(1-\alpha) g)(y)\right]=0 .
$$

Otherwise, if there exist $\delta>0$ such that for all $k$,

$$
(\alpha f+\lambda(1-\alpha) g)\left(X_{k}\right)-(\alpha f+\lambda(1-\alpha) g)(y)>\delta,
$$

then we have

$$
2 \sum_{k=0}^{\infty} \frac{a_{k}}{m}\left[(\alpha f+\lambda(1-\alpha) g)\left(X_{k}\right)-(\alpha f+\lambda(1-\alpha) g)(y)\right]>\frac{\delta}{m} \sum_{k=0}^{\infty} a_{k}=+\infty,
$$

which is impossible. 
Continuity of $\alpha f+\lambda(1-\alpha) g$ implies that all the limit points of $\left\{X_{k}\right\}$ are belong to $Y^{*}(\alpha, \lambda)$. Since $\left\{v_{i}\right\}$ is a dense subset of $Y^{*}$ and $\left\|X_{k}-v_{i}\right\|$ converges, it follows that $\left\{X_{k}\right\}$ cannot have more than one limit point, so it must converge to some vector $y \in Y^{*}(\alpha, \lambda)$. This completes the proof of Proposition 4.6.

Remark. In the proof above, we choose $y^{*}$ in $Y^{*}(\alpha, \lambda)$. With such a choice, there is no guarantee that the term

$$
2 \alpha \frac{a_{k}}{m}\left(f\left(X_{k}\right)-f\left(y^{*}\right)\right)-2(1-\alpha) \frac{b_{k}}{n}\left(g\left(X_{k}\right)-g\left(y^{*}\right)\right)
$$

is nonnegative for all $k$, as required in supermartingale convergence theorem. This is why we need Assumption 3.3 because we need to take the term $4 M\left|\frac{b_{k}}{n}-\lambda \frac{a_{k}}{m}\right|$ out of it.

Theorem 4.7. Let Assumptions 3.1, 3.3 and 4.5 hold. Assume that $(1-\alpha) \lambda=$ $\alpha$ and $\alpha \in(0,1)$. Then the sequence $\left\{X_{k}\right\}$ generated by RDA (9)-(11) converges to an optimal solution in $Y^{*}$ with probability 1 .

Proof. It follows from Proposition 4.6 and the definition of $Y^{*}(\alpha, \lambda)$, which coincides with $Y^{*}$ when the condition $(1-\alpha) \lambda=\alpha$ holds. This completes the proof.

Remark. Under the assumptions in Theorem 4.7, and also assume that the $\operatorname{limit}_{\lim _{k \rightarrow \infty}} \frac{b_{k}}{a_{k}}$ exists and equals 1 . Moreover, assume that $m=2 n$. Then $\lambda=2$ because $\lambda=\frac{m}{n} \lim _{k \rightarrow \infty} \frac{b_{k}}{a_{k}}$, by Proposition 4.10 in Section 4.4. Theorem 4.7 shows that the price process $\left\{X_{k}\right\}$ converges to a Walrasian equilibrium of the underlying economy, with probability 1 , if $\alpha=\frac{2}{3}$. Once $\lambda, n$ or $m$ has a change, we must also change $\alpha$ accordingly in order to achieve a Walrasian equilibrium. Otherwise the price process $\left\{X_{k}\right\}$ still converges but it may not converge to a Walrasian equilibrium of the underlying economy. Once again, Assumption 3.3 is the key. If it does not hold, then Theorem 4.7 fails again, as shown in Xu et al. $(2015,2016)$.

\subsection{A Numerical Example}

The following example has been also studied by Xu et al. $(2014,2015,2016)$. Now we use this example to illustrate why Theorems 4.4 and 4.7 may fail to 
converge to a Walrasian equilibrium of the underlying economy if the condition $\lambda=\frac{\alpha}{1-\alpha}$ does not hold. There are three sellers, $i=1,2,3$, with each seller $i=1,2,3$ an initial endowment of $(i+1)$ units of an identical (divisible) good. There are five buyers $j=1,2, \cdots, 5$, each buyer $j$ 's consumer's surplus or profit function $g_{j}: R_{+} \rightarrow R$ is obtained from

$$
g_{j}(y)=\max _{q \geq 0} u_{j}(q)-q y,
$$

where $u_{j}:[0, \infty) \rightarrow R_{+}$is $j$ 's utility function given by $u_{j}(q)=(j+1)+$ $2 \sqrt{(j+1) q}$. The supply curve for each seller is $S_{i}(y)=[0, i+1]$ for $y=0$ and $S_{i}(y)=i+1$ for $y>0, i=1,2,3$. The demand curve $D_{j}(y)=q_{j}^{*}$, where $u_{i}^{\prime}\left(q_{j}^{*}\right)=y$ for $y>>, j=1,2, \cdots, 5$. In this example, we can set $f_{i}(y)=$ $(i+1) y$ for $i \in I=\{1,2,3\}$ and $g_{j}(y)=(j+1)+\frac{j+1}{y}$ for $j \in J=\{1,2, \cdots, 5\}$ so that $D_{j}(y)=q_{j}^{*}=\frac{j+1}{y^{2}}$. Thus, the equilibrium price equals $y^{*}=\sqrt{\frac{20}{9}}=1.49$.

Note that the three sellers and five buyers have no knowledge where the equilibrium price 1.49 is. Neither do they know the total demand and supply. Each of them just submits their bid and ask based on their own private information. The two double auctions acting as a clearinghouse integrate individually "dispersed and incomplete information" (Hayek, 1945) into prices. While the equilibrium price equals $y^{*}=1.49$, the price, to which the price process under RDA converges, is $\sqrt{\frac{\lambda(1-\alpha)}{\alpha}} y^{*}$. Thus, if $\alpha=0.1$ and $\lambda=1$, the price process under RDA converges to the price $3 y^{*}, 200 \%$ higher than the original Walrasian equilibrium price $y^{*}$. If $\alpha=0.5$ while $\lambda=9$, the price process converges to $3 y^{*}$ as well. A crash price is also possible. For example, with $\alpha=0.8$ and $\lambda=1$, the price process converges to $\frac{1}{2} y^{*}, 50 \%$ lower than the equilibrium price of the original economy. Xu et al. $(2015,2016)$ provide simulations that are consistent with these theoretical predictions under Theorem 4.7.

For Theorem 4.4, we may assume that there are five sellers to the above example. Now the original Walrasian equilibrium becomes $y^{*}=1$. With $\alpha=0.1$ and $\lambda=1$, the CDA generates a sequence of prices that converges to $3,200 \%$ higher than the original equilibrium price $y^{*}=1$. Note that the initial distribution of endowments among the five agents is not that important.

Assume that $\lim _{k} \frac{b_{k}}{a_{k}}$ exists and equals 1 . There are some $\lambda$ such that the $\lambda$ conditions in Assumptions 3.2 and 3.3 hold. Then we know that $\lambda=$ $\lim _{k} \frac{b_{k}}{a_{k}}=1$ for CDA and RDA for five sellers and five buyers economy, the two auctions generate price sequences that converge to the equilibrium price 1 
when $\alpha=\frac{1}{2}$. Any other $\alpha$ can result in a price that is either higher or lower than the equilibrium price 1 .

Consider the three sellers and five buyers economy with RDA again. Also assume that $\lim _{k} \frac{b_{k}}{a_{k}}$ exists and equals 1 and there are some $\lambda$ such that Assumption 3.3 holds. Then $\lambda=\frac{3}{5}$. In order for RAD to achieve the equilibrium price 1.49 , one must have $\alpha$ equal $\frac{3}{8}$. Any other $\alpha$ will result in a price that is either higher or lower than the equilibrium price 1.49. Therefore, whether DA can achieve an equilibrium depends on a combination of $\alpha$ and $\lambda$ and the $\lambda$ condition.

\subsection{A Discussion about the $\lambda$ Condition}

We have seen that $\lambda$ plays a key role. In Assumption 3.2, we need $\lambda$ to satisfy the condition such that $\sum_{k=0}^{\infty}\left|b_{k}-\lambda a_{k}\right|<+\infty$. Is there such a $\lambda$ for any two sequences $\left\{a_{k}\right\}$ and $\left\{b_{k}\right\}$ that satisfy Assumption 3.1? Unfortunately the answer is not always affirmative. The relative strength between ask and bid step sizes is quite subtle for CDA or RDA mechanism.

\section{Example 4.8. Let}

$$
\begin{aligned}
& a_{k}= \begin{cases}\frac{1}{k}, & k \text { is odd } \\
\frac{1}{k^{2}}, & k \text { is even }\end{cases} \\
& b_{k}= \begin{cases}\frac{1}{k^{2}}, & k \text { is odd } \\
\frac{1}{k}, & k \text { is even }\end{cases}
\end{aligned}
$$

Then

$$
\begin{aligned}
\sum_{k=0}^{\infty}\left|a_{k}-\lambda b_{k}\right| & \geq \sum_{\substack{k \geq[\lambda]+1 \\
k \text { is odd }}}\left|a_{k}-\lambda b_{k}\right| \\
& =\sum_{\substack{k \geq[\lambda]+1 \\
k \text { is odd }}} a_{k}-\lambda \sum_{\substack{k \geq[\lambda]+1 \\
k \text { is odd }}} b_{k} \\
& =\sum_{\substack{k \geq[\lambda]+1 \\
k \text { is odd }}} \frac{1}{k}-\lambda \sum_{\substack{k \geq[\lambda]+1 \\
k \text { is odd }}} \frac{1}{k^{2}}=+\infty
\end{aligned}
$$


for any $\lambda$.

The next example shows that even if the $\operatorname{limit}_{\lim } \rightarrow \infty \frac{b_{k}}{a_{k}}$ exits and equals 1 , there may not exist $\lambda$ that satisfies Assumption 3.2.

Example 4.9. Let $a_{k}=\frac{1}{k^{\frac{3}{4}}}$ and $b_{k}=a_{k}\left(1+a_{k}^{\frac{1}{3}}\right)$. Then

$$
\lim _{k \rightarrow \infty} \frac{b_{k}}{a_{k}}=\lim _{k \rightarrow \infty}\left(1+\frac{1}{k^{\frac{1}{4}}}\right)=1 .
$$

But

$$
\sum_{k=0}^{\infty}\left|b_{k}-a_{k}\right|=\sum_{k=0}^{\infty} a_{k}^{\frac{4}{3}}=\sum_{k=0}^{\infty} \frac{1}{k}=+\infty .
$$

Note that, by Proposition 4.10 below, if there exists a $\lambda$ that satisfies Assumption 3.2, it can only be 1 . Hence, no $\lambda$ exists and satisfies Assumption 3.2 here.

The following answers what $\lambda$ must be.

Proposition 4.10. Let Assumption 3.1 hold. If $\sum_{k=0}^{\infty}\left|b_{k}-\lambda a_{k}\right|<+\infty$ for some $\lambda$, then the following must hold

$$
\liminf _{k \rightarrow \infty} \frac{b_{k}}{a_{k}} \leq \lambda \leq \limsup _{k \rightarrow \infty} \frac{b_{k}}{a_{k}}
$$

Proof. If there exist $\delta>0$ and $k_{0}$ such that $\frac{b_{k}}{a_{k}}-\lambda>\delta$ for all $k \geq k_{0}$, then

$$
\sum_{k=k_{0}}^{\infty}\left|b_{k}-\lambda a_{k}\right| \geq \delta \sum_{k=k_{0}}^{\infty} a_{k}=+\infty, \quad \text { a contradiction. }
$$

Hence, $\liminf _{k \rightarrow \infty} \frac{b_{k}}{a_{k}} \leq \lambda$ is one necessary condition for $\sum_{k=0}^{\infty}\left|b_{k}-\lambda a_{k}\right|<+\infty$. Similarly, $\lim \sup _{k \rightarrow \infty} \frac{b_{k}}{a_{k}} \geq \lambda$. This completes the proof.

Proposition 4.11. Let Assumption 3.1 hold. If there exists a $\lambda$ such that $\sum_{k=0}^{\infty}\left|b_{k}-\lambda a_{k}\right|<+\infty$, then it must be unique. 
Proof. Suppose, on the contrary, that there are two $\lambda$ and $\lambda^{\prime}$ such that

$$
\sum_{k=0}^{\infty}\left|b_{k}-\lambda a_{k}\right|<+\infty \text { and } \sum_{k=0}^{\infty}\left|b_{k}-\lambda^{\prime} a_{k}\right|<+\infty .
$$

Then

$$
\left|\lambda-\lambda^{\prime}\right| \sum_{k=0}^{\infty} a_{k} \leq \sum_{k=0}^{\infty}\left|b_{k}-\lambda a_{k}\right|+\sum_{k=0}^{\infty}\left|b_{k}-\lambda^{\prime} a_{k}\right|<+\infty .
$$

But $\sum_{k=0}^{\infty} a_{k}=+\infty$, a contradiction. This completes the proof.

Thus, there exists at most one $\lambda$ that satisfies Assumption 3.2 for any two given sequences $\left\{a_{k}\right\}$ and $\left\{b_{k}\right\}$ satisfying Assumption 3.1. But for any given $\lambda$, there are a family of step sizes $\left\{a_{k}\right\}$ and $\left\{b_{k}\right\}$ that satisfy Assumptions 3.1 and 3.2. Let $a_{k}=\frac{1}{k}$ and $b_{k}=2 a_{k}+c a_{k}^{2}$. Then Assumption 3.2 is satisfied with $\lambda=2$ for any positive finite number $c$ and any $\left\{a_{k}\right\}$ that satisfies Assumption 3.1 .

\section{DA MECHANISM WITH STOCHASTIC NOISES}

Let $f=\sum_{i=1}^{m} f_{i}$ and $g=\sum_{i=1}^{m} g_{i}$. Assume $X_{0}$ is a random initial vector. Let $\varepsilon_{i, k}$ and $\delta_{i, k}$ denote two independent random noise vectors. (DA) mechanism with stochastic noises is defined as follows.

Let $\Phi_{0, k}=X_{k}$. For $i=1,2, \cdots, m$, let

$$
\begin{aligned}
\psi_{i, k} & =\Phi_{i-1, k}-a_{k}\left(h_{i, k}+\varepsilon_{i, k}\right), h_{i, k} \in \partial f_{i}\left(\Phi_{i-1, k}\right) \\
\varphi_{i, k} & =\Phi_{i-1, k}-b_{k}\left(\ell_{i, k}+\delta_{i, k}\right), \ell_{i, k} \in \partial g_{i}\left(\Phi_{i-1, k}\right) \\
\Phi_{i, k} & =P_{Y}\left(\alpha \psi_{i, k}+(1-\alpha) \varphi_{i, k}\right), \quad \alpha \in[0,1] .
\end{aligned}
$$

Let

$$
X_{k+1}=\Phi_{m, k} .
$$

$P_{Y}$ is the Euclidean projection onto $Y$.

We define $\mathscr{F}_{k}^{i}$ to be the $\sigma$-algebra generated by the sequence

$$
\Phi_{0,0}, \Phi_{1,0}, \cdots, \Phi_{m, 0}, \cdots, \Phi_{i, k}
$$

Note that $\mathscr{F}_{k}^{0}$ is also denoted as $\mathscr{F}_{k}$. 
Assumption 5.1. There exist deterministic scalar sequences $\left\{\mu_{k}\right\},\left\{v_{k}\right\},\left\{\tau_{k}\right\}$ and $\left\{\sigma_{k}\right\}$ that satisfy the following inequalities for all $i$ and $k$ :

$$
\begin{aligned}
\left\|E\left[\varepsilon_{i, k} \mid \mathscr{F}_{k}^{i-1}\right]\right\| & \leq \mu_{k}, & & \left\|E\left[\delta_{i, k} \mid \mathscr{F}_{k}^{i-1}\right]\right\| \leq \tau_{k} ; \\
E\left[\left\|\varepsilon_{i, k}\right\|^{2} \mid \mathscr{F}_{k}^{i-1}\right] & \leq v_{k}^{2}, & & E\left[\left\|\delta_{i, k}\right\|^{2} \mid \mathscr{F}_{k}^{i-1}\right] \leq \sigma_{k}^{2} .
\end{aligned}
$$

Note that $\mu_{k} \leq v_{k}$ and $\tau_{k} \leq \sigma_{k}$ for all $k=0,1, \cdots$. The noise terms in the assumption above are similar to those in Ram et al. (2009). Their results, however, do not apply to (DA) mechanism (14)-(17) because there are two sequences of step-sizes that interact together to determine the price iteration process $\left\{X_{k}\right\}$.

The following lemma is the key and its proof is provided in the Appendix.

Lemma 5.2. Let Assumption 5.1 hold. Then the sequence $\left\{X_{k}\right\}$ generate by (DA) mechanism with stochastic noises (14)-(17) is such that for any step-size rule and any $y \in Y$,

$$
\begin{aligned}
E\left[\left\|X_{k+1}-y\right\|^{2} \mid \mathscr{F}_{k-1}^{m}\right] \leq & \left\|X_{k}-y\right\|^{2}-2 \alpha a_{k}\left(f\left(X_{k}\right)-f(y)\right) \\
& -2(1-\alpha) b_{k}\left(g\left(X_{k}\right)-g(y)\right) \\
& +2\left(\alpha a_{k} \mu_{k}+(1-\alpha) b_{k} \tau_{k}\right) \sum_{i=1}^{m} E\left[\left\|\Phi_{i-1, k}-y\right\| \| \mathscr{F}_{k-1}^{m}\right] \\
& +\left(\alpha a_{k} C+(1-\alpha) b_{k} D+\alpha m a_{k} v_{k}+(1-\alpha) m b_{k} \sigma_{k}\right)^{2},
\end{aligned}
$$

Note that $\mathscr{F}_{k-1}^{m}=\mathscr{F}_{k}^{0}$.

\subsection{Main Result with Stochastic Noises}

Assumption 5.3. The following holds:

$$
\sum_{k=0}^{\infty} a_{k} \mu_{k}<\infty, \sum_{k=0}^{\infty} b_{k} \tau_{k}<\infty, \sum_{k=0}^{\infty} a_{k}^{2} v_{k}^{2}<\infty, \sum_{k=0}^{\infty} b_{k}^{2} \sigma_{k}^{2}<\infty .
$$

Proposition 5.4. Let Assumptions 3.1, 3.2, 5.1 and 5.3 hold. Then the sequence $\left\{X_{k}\right\}$ generated by (DA) mechanism with stochastic noises (14)-(17) converges to an optimal solution $y^{*} \in Y^{*}(\alpha, \lambda)$, with probability 1 . 
Proof. By Lemma 5.2, for any $y^{*} \in Y^{*}(\alpha, \lambda)$, we have that

$$
\begin{aligned}
& E\left[\left\|X_{k+1}-y^{*}\right\|^{2} \mid \mathscr{F}_{k-1}^{m}\right] \leq\left\|X_{k}-y^{*}\right\|^{2}+M_{k} \\
& -2 \alpha a_{k}\left(f\left(X_{k}\right)-f\left(y^{*}\right)\right)-2(1-\alpha) b_{k}\left(g\left(X_{k}\right)-g\left(y^{*}\right)\right) \\
& +2\left(\alpha a_{k} \mu_{k}+(1-\alpha) b_{k} \tau_{k}\right) \sum_{i=1}^{m} E\left[\left\|\Phi_{i-1, k}-y^{*}\right\| \mid \mathscr{F}_{k-1}^{m}\right],
\end{aligned}
$$

where $M_{k}=\left(\alpha a_{k} C+(1-\alpha) b_{k} D+m \alpha a_{k} v_{k}+m(1-\alpha) b_{k} \sigma_{k}\right)^{2}$.

Since

$$
\begin{aligned}
E\left[\left\|\Phi_{i-1, k}-y^{*}\right\| \mid \mathscr{F}_{k-1}^{m}\right] \leq & E\left[\left\|\Phi_{i-1, k}-X_{k}\right\| \mid \mathscr{F}_{k-1}^{m}\right]+\left\|X_{k}-y^{*}\right\| \\
\leq & \sum_{j=1}^{i-1}\left(\alpha a_{k} C_{j}+(1-\alpha) b_{k} D_{j}+\alpha a_{k} v_{k}\right. \\
& \left.+(1-\alpha) b_{k} \sigma_{k}\right)+\left\|X_{k}-y^{*}\right\| .
\end{aligned}
$$

In the second inequality above we have used Lemma 5.2.1 in the Appendix and Assumption 5.1.

Hence,

$$
\begin{aligned}
2\left(\alpha a_{k} \mu_{k}+\right. & \left.(1-\alpha) b_{k} \tau_{k}\right) \sum_{i=1}^{m} E\left[\left\|\Phi_{i-1, k}-y^{*}\right\| \mid \mathscr{F}_{k-1}^{m}\right] \\
\leq & 2\left(\alpha a_{k} \mu_{k}+(1-\alpha) b_{k} \tau_{k}\right) \sum_{i=1}^{m}\left\{\sum _ { j = 1 } ^ { i - 1 } \left(\alpha a_{k} C_{j}\right.\right. \\
& \left.\left.+(1-\alpha) b_{k} D_{j}+\alpha a_{k} v_{k}+(1-\alpha) b_{k} \sigma_{k}\right)+\left\|X_{k}-y^{*}\right\|\right\} \\
\leq & 2\left(\alpha a_{k} \mu_{k}+(1-\alpha) b_{k} \tau_{k}\right) \sum_{i=1}^{m} \sum_{j=1}^{i-1}\left\{\alpha a_{k} C_{j}\right. \\
& \left.+(1-\alpha) b_{k} D_{j}+\alpha a_{k} v_{k}+(1-\alpha) b_{k} \sigma_{k}\right\} \\
& +m\left(\alpha a_{k} \mu_{k}+(1-\alpha) b_{k} \tau_{k}\right)\left(\left\|X_{k}-y^{*}\right\|^{2}+1\right) .
\end{aligned}
$$

In the last inequality above we have used the inequality $a^{2}+1 \geq 2 a$.

And then

$$
\begin{aligned}
E\left[\left\|X_{k+1}-y^{*}\right\|^{2} \mid \mathscr{F}_{k-1}^{m}\right] \leq & \left(1+m\left(\alpha a_{k} \mu_{k}+(1-\alpha) b_{k} \tau_{k}\right)\right)\left\|X_{k}-y^{*}\right\|^{2} \\
& +\left(M_{k}+N_{k}\right)-2 \alpha a_{k}\left(f\left(X_{k}\right)-f\left(y^{*}\right)\right) \\
& -2(1-\alpha) b_{k}\left(g\left(X_{k}\right)-g\left(y^{*}\right)\right)
\end{aligned}
$$


where

$$
\begin{aligned}
N_{k}= & 2\left(\alpha a_{k} \mu_{k}+(1-\alpha) b_{k} \tau_{k}\right) \sum_{i=1}^{m} \sum_{j=1}^{i-1}\left\{\alpha a_{k} C_{j}+(1-\alpha) b_{k} D_{j}\right. \\
& \left.+\alpha a_{k} \nu_{k}+(1-\alpha) b_{k} \sigma_{k}\right\}+m\left(\alpha a_{k} \mu_{k}+(1-\alpha) b_{k} \tau_{k}\right) .
\end{aligned}
$$

We need the following lemma in our proof below.

Lemma 3.2 in Ram et al. (2009): Let $(\Omega, \mathscr{F}, P)$ be a probability space and let $\mathscr{F}_{0} \subset \mathscr{F}_{1} \subset \cdots$ be a sequence of sub $\sigma$-fields of $\mathscr{F}$. Let $u_{k}, v_{k}$ and $w_{k}$, $k=0,1,2, \cdots$, be non-negative $\mathscr{F}_{k}$-measurable random variables and let $\left\{q_{k}\right\}$ be a deterministic sequence. Assume that $\sum_{k=0}^{\infty} q_{k}<\infty, \sum_{k=0}^{\infty} w_{k}<\infty$, and

$$
E\left\{u_{k+1} \mid \mathscr{F}_{k}\right\} \leq\left(1+q_{k}\right) u_{k}-v_{k}+w_{k}
$$

hold with probability 1 . Then, with probability 1 , the sequence $\left\{u_{k}\right\}$ converges to a non-negative random variable and $\sum_{k=0}^{\infty} v_{k}<\infty$.

To apply Lemma 3.2 in Ram et al. (2009), let $q_{k}=m\left(\alpha a_{k} \mu_{k}+(1-\alpha) b_{k} \tau_{k}\right)$ and $W_{k}=M_{k}+N_{k}$.

Then

$$
\begin{aligned}
\sum_{k=0}^{\infty} q_{k} & =m \alpha \sum_{k=0}^{\infty} a_{k} \mu_{k}+m(1-\alpha) \sum_{k=0}^{\infty} b_{k} \tau_{k}<+\infty \\
\sum_{k=0}^{\infty} W_{k} & =\sum_{k=0}^{\infty}\left(M_{k}+N_{k}\right) .
\end{aligned}
$$

Since, using $a^{2}+b^{2} \geq 2 a b$ and Assumption 5.3,

$$
\begin{aligned}
\sum_{k=0}^{\infty} M_{k} & =\sum_{k=0}^{\infty}\left(\alpha a_{k} C+(1-\alpha) b_{k} D+m \alpha a_{k} v_{k}+m(1-\alpha) b_{k} \sigma_{k}\right)^{2} \\
& \leq 4 \sum_{k=0}^{\infty}\left[\left(\alpha a_{k} C\right)^{2}+\left((1-\alpha) b_{k} D\right)^{2}+\left(m \alpha a_{k} v_{k}\right)^{2}+\left(m(1-\alpha) b_{k} \sigma_{k}\right)^{2}\right] \\
& <\infty
\end{aligned}
$$


and, by $\mu_{k} \leq v_{k}$ and $\tau_{k} \leq \sigma_{k}$ in Assumption 5.1,

$$
\begin{aligned}
\sum_{k=0}^{\infty} N_{k}= & \sum_{k=0}^{\infty} 2\left(\alpha a_{k} \mu_{k}+(1-\alpha) b_{k} \tau_{k}\right) \sum_{i=1}^{m} \sum_{j=1}^{i-1}\left\{\alpha a_{k} C_{j}\right. \\
& \left.+(1-\alpha) b_{k} D_{j}+\alpha a_{k} v_{k}+(1-\alpha) b_{k} \sigma_{k}\right\}+\sum_{k=0}^{\infty} q_{k} \\
\leq & \sum_{k=0}^{\infty}\left[\sum_{i=1}^{m}\left(\alpha a_{k} v_{k}+(1-\alpha) b_{k} \sigma_{k}+\alpha a_{k} C_{i}+(1-\alpha) b_{k} D_{i}\right)\right]^{2}+\sum_{k=0}^{\infty} q_{k} \\
\leq & \sum_{k=0}^{\infty} M_{k}+\sum_{k=0}^{\infty} q_{k}<\infty
\end{aligned}
$$

we have that $\sum_{k=0}^{\infty} W_{k}<\infty$.

Therefore, we get, with probability 1 , the sequence $\left\|X_{k}-y^{*}\right\|^{2}$ converges to some non-negative random variable for every $y^{*} \in Y(\alpha, \lambda)$. Also with probability 1 , we have

$$
\sum_{k=0}^{\infty}\left(\alpha a_{k}\left(f\left(X_{k}\right)-f\left(y^{*}\right)\right)+(1-\alpha) b_{k}\left(g\left(X_{k}\right)-g\left(y^{*}\right)\right)\right)<+\infty,
$$

which implies that

$$
\begin{aligned}
& \sum_{k=0}^{\infty} a_{k}\left[(\alpha f+(1-\alpha) \lambda g)\left(X_{k}\right)-(\alpha f+(1-\alpha) \lambda g)\left(y^{*}\right)\right] \\
& \leq \sum_{k=0}^{\infty}\left(\alpha a_{k}\left(f\left(X_{k}\right)-f\left(y^{*}\right)\right)+(1-\alpha) b_{k}\left(g\left(X_{k}\right)-g\left(y^{*}\right)\right)\right) \\
& +\sum_{\substack{k=0 \\
<}}^{\infty}(1-\alpha)\left|b_{k}-\lambda a_{k}\right|\left|g\left(X_{k}\right)-g\left(y^{*}\right)\right| \\
& <+\infty .
\end{aligned}
$$

Since $Y$ is compact and $g$ is continuous, the image of $g$ is bounded. Assume $\exists M>0$ such that $|g(y)| \leq M$ for all $y \in Y$. Then

$$
\sum_{k=0}^{\infty}(1-\alpha)\left|b_{k}-\lambda a_{k}\right|\left|g\left(X_{k}\right)-g\left(y^{*}\right)\right| \leq 2 M(1-\alpha) \sum_{k=0}^{\infty}\left|b_{k}-\lambda a_{k}\right|<+\infty .
$$

Since $\sum_{k=0}^{\infty} a_{k}=+\infty$, then

$$
\liminf _{k \rightarrow \infty}(\alpha f+(1-\alpha) \lambda g)\left(X_{k}\right)=(\alpha f+(1-\alpha) \lambda g)\left(y^{*}\right),
$$


with probability 1 .

By considering a sample path for which

$$
\liminf _{k \rightarrow \infty}(\alpha f+(1-\alpha) \lambda g)\left(X_{k}\right)=(\alpha f+(1-\alpha) \lambda g)\left(y^{*}\right)
$$

and $\left\|X_{k}-y^{*}\right\|^{2}$ converges for any $y^{*}$, we conclude that the sample sequence must converge to some $y^{*}$ in view of continuity of $f$. Hence, the sequence $\left\{X_{k}\right\}$ converges to some optimal solution in $Y^{*}(\alpha, \lambda)$ with probability 1 . This completes the proof of Proposition 5.4x .

Remark. Strictly speaking, the term $2 \alpha a_{k}\left(f\left(X_{k}\right)-f\left(y^{*}\right)\right)+2(1-\alpha) b_{k}[$ $\left.g\left(X_{k}\right)-g\left(y^{*}\right)\right]$ may not be nonnegative so that we cannot directly apply Lemma 3.2. But this will not cause a problem as long as Assumptions 3.1 and 3.2 are satisfied because we can always follow the proof of Proposition 4.6 to remove the term $4 M\left|\frac{b_{k}}{n}-\lambda \frac{a_{k}}{m}\right|$ from it.

Immediately we obtain from Proposition 5.4 the following.

Theorem 5.5. Let Assumptions 3.1, 3.2, 5.1 and 5.3 hold. Assume that $\lambda=\frac{\alpha}{1-\alpha}, \alpha \in(0,1)$. Then the sequence $\left\{X_{k}\right\}$ generated by the DA mechanism with stochastic noises (14)-(17) converges to an optimal solution in $Y^{*}$, with probability 1 .

\subsection{RDA Mechanism with Stochastic Noises}

Recall that $w_{k}$ is a random variable taking equiprobable values from the set $\{1,2, \cdots, m\}$ and $w_{k}^{\prime}$ is a random variable taking equiprobable values from the set $\{1,2, \cdots, n\}$. Also recall that $h_{w_{k}}\left(X_{k}\right) \in \partial f_{w_{k}}\left(X_{k}\right)$ and $\ell_{w_{k}^{\prime}}\left(X_{k}\right) \in \partial g_{w_{k}^{\prime}}\left(X_{k}\right)$, where if $w_{k}$ takes a value $j$, then the vector $\partial f_{w_{k}}\left(X_{k}\right)$ is $\partial f_{j}\left(X_{k}\right)$, similarly for $g$.

Our sequence $\left\{X_{k}\right\}$ is generated by (RDA) mechanism with stochastic noises as below.

Given $X_{k}$, let

$$
\psi_{k+1}=X_{k}-a_{k}\left(h_{w_{k}}\left(X_{k}\right)+\varepsilon_{w_{k}, k}\right), \quad h_{w_{k}}\left(X_{k}\right) \in \partial f_{w_{k}}\left(X_{k}\right)
$$

and

$$
\varphi_{k+1}=X_{k}-b_{k}\left(\ell_{w_{k}^{\prime}}\left(X_{k}\right)+\delta_{w_{k}^{\prime}, k}\right), \quad \ell_{w_{k}^{\prime}}\left(X_{k}\right) \in \partial g_{w_{k}^{\prime}}\left(X_{k}\right) .
$$


And set

$$
X_{k+1}=P_{Y}\left(\alpha \psi_{k+1}+(1-\alpha) \varphi_{k+1}\right), \quad \alpha \in[0,1] .
$$

$P_{Y}$ is the Euclidean projection onto $Y$.

We define $\mathscr{F}_{k}$ to be the $\sigma$-field generated by $X_{0}, X_{1}, \cdots, X_{k}$.

Assumption 5.8. The sequence $\left\{w_{k}\right\}\left(\left\{w_{k}^{\prime}\right\}\right)$ is a sequence of independent random variables, each uniformly distributed over the set $\{1,2, \cdots, m\}(\{1,2, \cdots, n\})$. Furthermore, the two sequences $\left\{w_{k}\right\}$ and $\left\{w_{k}^{\prime}\right\}$ are independent of the sequence $\left\{X_{k}\right\}$.

Proposition 5.9. Let Assumptions 3.1, 3.3, 5.1, 5.3, and 5.8 hold. Then the sequence $\left\{X_{k}\right\}$ generated by (RDA) with randomization and stochastic noises (18)-(20) converges to an optimal solution in $Y^{*}(\alpha, \lambda)$, with probability 1 .

Proof. The proof is similar to those of Proposition 4.6 and Proposition 5.4 and thus omitted.

The following result follows from Proposition 5.9 and the definition of $Y^{*}(\alpha, \lambda)$, which is the same as $Y^{*}$ when the equality $\lambda=\frac{\alpha}{1-\alpha}$ holds.

Theorem 5.10. Let Assumptions 3.1, 3.3, 5.1, 5.3, and 5.8 hold. Assume that $\lambda=\frac{\alpha}{1-\alpha}, \alpha \in(0,1)$. Then the sequence $\left\{X_{k}\right\}$ generated by (RDA) mechanism with stochastic noises (18)-(20) converges to an optimal solution in $Y^{*}$, with probability 1 .

\section{CONCLUDING REMARKS}

This paper studies two dynamic double auctions and examines the question of whether the price processes they generate can converge to a Walrasian equilibrium of the underlying economy. We show that the weight $\alpha$ and the $\lambda$ condition are important for the convergence of these price processes. With the right combinations of $\alpha$ and $\lambda$, the price processes generated by the two auctions converge to a Walrasian equilibrium of the underlying economy. If the combination is not right, the price processes may generate a bubble or 
crash. Numerical examples show that such a bubble or crash can reach an enormous level, as shown in Xu et al. (2015, 2016), which provide extensions of the convergence results presented in $\mathrm{Ma} \& \mathrm{Li}$ (2011) to more complicated environments. Our results and those in the literature imply that the form of double auctions does matter very much for the price determination of an asset or a good traded in an exchange market. Because human emotion such as fear and greed may affect the two parameters, our results also shed some important light on how human emotion may impact the price of an asset in an exchange market that uses double auctions as clearinghouses.

In a laissez-faire economy, private information can be successively incorporated into the price of a good through individual decisions of what to buy or sell. Without knowing what may be the price at equilibrium for a good, the market via an invisible hand can reach an equilibrium. Such a view is the foundation for economic analyses based on equilibrium. The incremental subgradient method in Nedić \& Bertsekas (2001) can be used to show how this may be done in theory for a quasilinear economy, with some intervention from a central authority by setting the step size rules properly. Such an approach is especially important for market mechanisms since every individual has a piece of private information while the market equilibrium prices must reflect all relevant private information. Chen et al. (2016) provide a different approach for a totally uncoordinated and decentralized market, in which every firm and every worker can form a matching pair randomly and seek opportunities to improve their individual positions. They prove that without any clearinghouse or coordination, starting with any matching and any salary scheme system, stable or not, a natural decentralized random matching process converges to a Walrasian equilibrium with probability one in finite time. It remains open to question if there is a good way to integrate the two approaches.

The study of the competitive efficiency of a DA mechanism started with experiments for an identical good in V. L. Smith $(1962,1965)$ where an artificial market was created with competitive equilibrium unknown to the buyers and sellers. In these experiments the DA mechanism converged quickly to a neighbor of the competitive equilibrium, even with a few participants. A great number of experiments have been conducted since then and a similar result has been obtained (Friedman \& Rust, 1993). In recent years the competitive efficiency of a DA mechanism has been retested in experiments with more complicated environments, which are deliberately designed to be a proxy of an exchange market. V. L. Smith et al. (1988) show that both bubbles and crashes 
can be generated by a DA mechanism under these environments. Therefore, the allocative efficiency of a DA mechanism is a complicated issue. We show in this paper that the efficiency of our two DA mechanisms depends on the relative strength of the two step-sizes of the bid and the ask through a parameter $\lambda$ and the weight how these bids and asks enter the price process. The two parameters may be considered as two "steering" factors because they act just like a steering in a vehicle; a different combination of the two directs the price process to different places.

Our study of the two DA mechanisms is applicable to a market where there are potentially a large number of agents and a large number of assets. The primary task of our paper is to provide an explanation of the price determination of a good. Our results are closely related to those obtained with the incremental subgradient method in Nedić \& Bertsekas (2001) and Ram et al. (2009). Because the problem $\mathscr{P}$ has so many other applications (Bertsekas, 2009, 2012), our DA mechanisms provide an alternative explanation of how an optimal solution can be approached for those environments (e.g., distributed and neural networks). 


\section{APPENDIX}

This appendix contains the proof of Lemma 5.2 which is divided into several steps.

Proof of Lemma 5.2. By non-expansive property of projection,

$$
\begin{aligned}
\left\|\Phi_{i, k}-y\right\|^{2} \leq & \left\|\alpha \psi_{i, k}+(1-\alpha) \varphi_{i, k}-y\right\|^{2} \\
= & \left\|\alpha\left(\psi_{i, k}-y\right)+(1-\alpha)\left(\varphi_{i, k}-y\right)\right\|^{2} \\
= & \alpha^{2}\left\|\psi_{i, k}-y\right\|^{2}+(1-\alpha)^{2}\left\|\varphi_{i, k}-y\right\|^{2}+2 \alpha(1-\alpha)\left\langle\psi_{i, k}-y, \varphi_{i, k}-y\right\rangle \\
= & \alpha^{2}\left\|\Phi_{i-1, k}-y-a_{k} h_{i, k}-a_{k} \varepsilon_{i, k}\right\|^{2} \\
& +(1-\alpha)^{2}\left\|\Phi_{i-1, k}-y-b_{k} \ell_{i, k}-b_{k} \delta_{i, k}\right\|^{2} \\
& +2 \alpha(1-\alpha)\left\langle\Phi_{i-1, k}-y-a_{k} h_{i, k}-a_{k} \varepsilon_{i, k}, \Phi_{i-1, k}-y-b_{k} \ell_{i, k}-b_{k} \delta_{i, k}\right\rangle \\
= & \alpha^{2}\left\|\Phi_{i-1, k}-y-a_{k} h_{i, k}\right\|^{2}+\alpha^{2} a_{k}^{2}\left\|\varepsilon_{i, k}\right\|^{2} \\
& -2 \alpha^{2}\left\langle\Phi_{i-1, k}-y-a_{k} h_{i, k}, a_{k} \varepsilon_{i, k}\right\rangle+2 \alpha(1-\alpha) a_{k} b_{k}\left\langle\varepsilon_{i, k}, \delta_{i, k}\right\rangle \\
& +(1-\alpha)^{2}\left\|\Phi_{i-1, k}-y-b_{k} \ell_{i, k}\right\|^{2}+(1-\alpha)^{2} b_{k}^{2}\left\|\delta_{i, k}\right\|^{2} \\
& -2(1-\alpha)^{2}\left\langle\Phi_{i-1, k}-y-b_{k} \ell_{i, k}, b_{k} \delta_{i, k}\right\rangle \\
& +2 \alpha(1-\alpha)\left\langle\Phi_{i-1, k}-y-a_{k} h_{i, k}, \Phi_{i-1, k}-y-b_{k} \ell_{i, k}\right\rangle \\
& -2 \alpha(1-\alpha)\left\langle\Phi_{i-1, k}-y-a_{k} h_{i, k}, b_{k} \delta_{i, k}\right\rangle \\
& -2 \alpha(1-\alpha)\left\langle\Phi_{i-1, k}-y-b_{k} \ell_{i, k}, a_{k} \varepsilon_{i, k}\right\rangle \\
= & \left\|\alpha\left(\Phi_{i-1, k}-y-a_{k} h_{i, k}\right)+(1-\alpha)\left(\Phi_{i-1, k}-y-b_{k} \ell_{i, k}\right)\right\|^{2} \\
& +\left\|\alpha a_{k} \varepsilon_{i, k}+(1-\alpha) b_{k} \delta_{i, k}\right\|^{2} \\
& -2 \alpha\left\langle\Phi_{i-1, k}-y-a_{k} h_{i, k}, \alpha a_{k} \varepsilon_{i, k}+(1-\alpha) b_{k} \delta_{i, k}\right\rangle \\
& -2(1-\alpha)\left\langle\Phi_{i-1, k}-y-b_{k} \ell_{i, k}, \alpha a_{k} \varepsilon_{i, k}+(1-\alpha) b_{k} \delta_{i, k}\right\rangle \\
= & \left\|\Phi_{i-1, k}-y\right\|^{2}-2 \alpha a_{k}\left\langle h_{i, k},\left(\Phi_{i-1, k}-y\right)\right\rangle \\
& -2(1-\alpha) b_{k}\left\langle\ell_{i, k},\left(\Phi_{i-1, k}-y\right)\right\rangle \\
& +\left\|\alpha a_{k} h_{i, k}+(1-\alpha) b_{k} \ell_{i, k}\right\|^{2}+\left\|\alpha a_{k} \varepsilon_{i, k}+(1-\alpha) b_{k} \delta_{i, k}\right\|^{2} \\
& -2 \alpha\left\langle\Phi_{i-1, k}-y-a_{k} h_{i, k}, \alpha a_{k} \varepsilon_{i, k}+(1-\alpha) b_{k} \delta_{i, k}\right\rangle \\
& -2(1-\alpha)\left\langle\Phi_{i-1, k}-y-b_{k} \ell_{i, k}, \alpha a_{k} \varepsilon_{i, k}+(1-\alpha) b_{k} \delta_{i, k}\right\rangle \\
& -2(1-\alpha)
\end{aligned}
$$

Taking conditional expectations with respect to the $\sigma$-field $\mathscr{F}_{k}^{i-1}$ leads to 


$$
\begin{aligned}
E\left[\left\|\Phi_{i, k}-y\right\|^{2} \mid \mathscr{F}_{k}^{i-1}\right] \leq & \left\{\left\|\Phi_{i-1, k}-y\right\|^{2}-2 \alpha a_{k}\left\langle h_{i, k},\left(\Phi_{i-1, k}-y\right)\right\rangle\right. \\
& \left.-2(1-\alpha) b_{k}\left\langle\ell_{i, k},\left(\Phi_{i-1, k}-y\right)\right\rangle+\left\|\alpha a_{k} h_{i, k}+(1-\alpha) b_{k} \ell_{i, k}\right\|^{2}\right\} \\
& +\left\{E\left[\left\|\alpha a_{k} \varepsilon_{i, k}+(1-\alpha) b_{k} \delta_{i, k}\right\|^{2} \mid \mathscr{F}_{k}^{i-1}\right]\right. \\
& -2 \alpha\left\langle\Phi_{i-1, k}-y-a_{k} h_{i, k}, E\left[\alpha a_{k} \varepsilon_{i, k}+(1-\alpha) b_{k} \delta_{i, k} \mid \mathscr{F}_{k}^{i-1}\right]\right\rangle \\
& -2(1-\alpha)\left\langle\Phi_{i-1, k}-y-b_{k} \ell_{i, k},\right. \\
& \left.\left.E\left[\alpha a_{k} \varepsilon_{i, k}+(1-\alpha) b_{k} \delta_{i, k} \mid \mathscr{F}_{k}^{i-1}\right]\right\rangle\right\} \\
= & I+I I .
\end{aligned}
$$

Consider II first. We have that, by Assumption 5.1,

$$
\begin{aligned}
I I \leq & \left(\alpha a_{k} v_{k}+(1-\alpha) b_{k} \sigma_{k}\right)^{2}+2 \alpha\left(\left\|\Phi_{i-1, k}-y\right\|+a_{k}\left\|h_{i, k}\right\|\right)\left(\alpha a_{k} \mu_{k}+(1-\alpha) b_{k} \tau_{k}\right) \\
& +2(1-\alpha)\left(\left\|\Phi_{i-1, k}-y\right\|+b_{k}\left\|\ell_{i, k}\right\|\right)\left(\alpha a_{k} \mu_{k}+(1-\alpha) b_{k} \tau_{k}\right) \\
= & \left(\alpha a_{k} v_{k}+(1-\alpha) b_{k} \sigma_{k}\right)^{2}+2\left\|\Phi_{i-1, k}-y\right\|\left(\alpha a_{k} \mu_{k}+(1-\alpha) b_{k} \tau_{k}\right) \\
& +2 \alpha a_{k} C_{i}\left(\alpha a_{k} \mu_{k}+(1-\alpha) b_{k} \tau_{k}\right)+2(1-\alpha) b_{k} D_{i}\left(\alpha a_{k} \mu_{k}+(1-\alpha) b_{k} \tau_{k}\right) .
\end{aligned}
$$

Now consider I. Since $h_{i, k} \in \partial f_{i}\left(\Phi_{i-1, k}\right)$ and $\ell_{i, k} \in \partial g_{i}\left(\Phi_{i-1, k}\right)$ so that

$$
\left\langle h_{i, k},\left(y-\Phi_{i-1, k}\right)\right\rangle \leq f_{i}(y)-f_{i}\left(\Phi_{i-1, k}\right)
$$

and

$$
\left\langle\ell_{i, k},\left(y-\Phi_{i-1, k}\right)\right\rangle \leq g_{i}(y)-g_{i}\left(\Phi_{i-1, k}\right),
$$

we have that

$$
\begin{aligned}
I \leq & \left\|\Phi_{i-1, k}-y\right\|^{2}-2 \alpha a_{k}\left(f_{i}\left(\Phi_{i-1, k}\right)-f_{i}(y)\right)-2(1-\alpha) b_{k}\left(g_{i}\left(\Phi_{i-1, k}\right)-g_{i}(y)\right) \\
& +\left\|\alpha a_{k} C_{i}+(1-\alpha) b_{k} D_{i}\right\|^{2} .
\end{aligned}
$$

Taking the expectations conditional on $\mathscr{F}_{k-1}^{m}=\mathscr{F}_{k}^{0}$, we obtain from $I+I I$ that

$$
\begin{aligned}
E\left[\left\|\Phi_{i, k}-y\right\|^{2} \mid \mathscr{F}_{k-1}^{m}\right] \leq & E\left[\left\|\Phi_{i-1, k}-y\right\|^{2} \mid \mathscr{F}_{k-1}^{m}\right]-2 \alpha a_{k}\left(f_{i}\left(X_{k}\right)-f_{i}(y)\right) \\
& -2(1-\alpha) b_{k}\left(g_{i}\left(X_{k}\right)-g_{i}(y)\right) \\
& +2 E\left[\left\|\Phi_{i-1, k}-y\right\| \mid \mathscr{F}_{k-1}^{m}\right]\left(\alpha a_{k} \mu_{k}+(1-\alpha) b_{k} \tau_{k}\right)+M_{i, k},
\end{aligned}
$$

where

$$
\begin{aligned}
M_{i, k}= & \left(\alpha a_{k} C_{i}+(1-\alpha) b_{k} D_{i}\right)^{2}+\left(\alpha a_{k} v_{k}+(1-\alpha) b_{k} \sigma_{k}\right)^{2} \\
& +2 \alpha a_{k} C_{i}\left(\alpha a_{k} \mu_{k}+(1-\alpha) b_{k} \tau_{k}\right)+2(1-\alpha) b_{k} D_{i}\left(\alpha a_{k} \mu_{k}+(1-\alpha) b_{k} \tau_{k}\right) \\
& +2 \alpha a_{k} E\left[\left\|f_{i}\left(\Phi_{i-1, k}\right)-f_{i}\left(X_{k}\right)\right\| \mid \mathscr{F}_{k-1}^{m}\right] \\
& +2(1-\alpha) b_{k} E\left[\left\|g_{i}\left(\Phi_{i-1, k}\right)-g_{i}\left(X_{k}\right)\right\| \mid \mathscr{F}_{k-1}^{m}\right] .
\end{aligned}
$$


Note that $\Phi_{0, k}=X_{k}$ and $\Phi_{m, k}=X_{k+1}$. Taking sum over $i=1,2, \cdots, m$, we have that

$$
\begin{aligned}
E\left[\left\|X_{k+1}-y\right\|^{2} \mid \mathscr{F}_{k-1}^{m}\right] \leq & \left\|X_{k}-y\right\|^{2}-2 \alpha a_{k}\left(f\left(X_{k}\right)-f(y)\right) \\
& -2(1-\alpha) b_{k}\left(g\left(X_{k}\right)-g(y)\right)+\sum_{i=1}^{m} M_{i, k} \\
& +2\left(\alpha a_{k} \mu_{k}+(1-\alpha) b_{k} \tau_{k}\right) \sum_{i=1}^{m} E\left[\left\|\Phi_{i-1, k}-y\right\| \mathscr{F}_{k-1}^{m}\right] .
\end{aligned}
$$

Next we consider $\sum_{i=1}^{m} M_{i, k}$.

Lemma 5.2.1. We claim that

$$
\left\|\Phi_{i-1, k}-X_{k}\right\| \leq \sum_{j=1}^{i-1}\left[\alpha a_{k} C_{j}+(1-\alpha) b_{k} D_{j}+\alpha a_{k}\left\|\varepsilon_{j, k}\right\|+(1-\alpha) b_{k}\left\|\delta_{j, k}\right\|\right] .
$$

Proof of Lemma 5.2.1. We prove by induction.

$$
\begin{aligned}
\left\|\Phi_{i, k}-X_{k}\right\| \leq & \left\|\left(\alpha \psi_{i, k}+(1-\alpha) \varphi_{i, k}\right)-X_{k}\right\| \\
\leq & \alpha\left\|\psi_{i, k}-X_{k}\right\|+(1-\alpha)\left\|\varphi_{i, k}-X_{k}\right\| \\
= & \alpha\left\|\Phi_{i-1, k}-a_{k} h_{i, k}-a_{k} \varepsilon_{i, k}-X_{k}\right\| \\
& +(1-\alpha)\left\|\Phi_{i-1, k}-b_{k} \ell_{i, k}-b_{k} \delta_{i, k}-X_{k}\right\| \\
\leq & \left\|\Phi_{i-1, k}-X_{k}\right\|+\alpha a_{k}\left\|h_{i, k}\right\|+(1-\alpha) b_{k}\left\|l_{i, k}\right\| \\
& +\alpha a_{k}\left\|\varepsilon_{i, k}\right\|+(1-\alpha) b_{k}\left\|\delta_{i, k}\right\| .
\end{aligned}
$$

By induction, we get that

$$
\left\|\Phi_{i, k}-X_{k}\right\| \leq \sum_{j=1}^{i}\left[\alpha a_{k} C_{j}+(1-\alpha) b_{k} D_{j}+\alpha a_{k}\left\|\varepsilon_{j, k}\right\|+(1-\alpha) b_{k}\left\|\delta_{j, k}\right\|\right] .
$$

This completes the proof of Lemma 5.2.1.

We now continue the proof of Lemma 5.2 and have

$$
\begin{aligned}
E\left[\left\|f_{i}\left(\Phi_{i-1, k}\right)-f_{i}\left(X_{k}\right)\right\| \mid \mathscr{F}_{k-1}^{m}\right] \leq & E\left[C _ { i } \sum _ { j = 1 } ^ { i - 1 } \left(\alpha a_{k} C_{j}+(1-\alpha) b_{k} D_{j}+\alpha a_{k}\left\|\varepsilon_{j, k}\right\|\right.\right. \\
& \left.\left.+(1-\alpha) b_{k}\left\|\delta_{j, k}\right\|\right) \mid \mathscr{F}_{k-1}^{m}\right] \\
\leq & C_{i} \sum_{j=1}^{i-1}\left\{\alpha a_{k} C_{j}+(1-\alpha) b_{k} D_{j}+\alpha a_{k} v_{k}\right. \\
& \left.+(1-\alpha) b_{k} \sigma_{k}\right\}
\end{aligned}
$$


and

$$
\begin{aligned}
E\left[\left\|g_{i}\left(\Phi_{i-1, k}\right)-g_{i}\left(X_{k}\right)\right\| \mid \mathscr{F}_{k-1}^{m}\right] \leq & E\left[D _ { i } \sum _ { j = 1 } ^ { i - 1 } \left\{\alpha a_{k} C_{j}+(1-\alpha) b_{k} D_{j}+\alpha a_{k}\left\|\varepsilon_{j, k}\right\|\right.\right. \\
& \left.\left.+(1-\alpha) b_{k}\left\|\delta_{j, k}\right\|\right\} \mid \mathscr{F}_{k-1}^{m}\right] \\
\leq & D_{i} \sum_{j=1}^{i-1}\left\{\alpha a_{k} C_{j}+(1-\alpha) b_{k} D_{j}+\alpha a_{k} v_{k}\right. \\
& \left.+(1-\alpha) b_{k} \sigma_{k}\right\} .
\end{aligned}
$$

Then

$$
\begin{aligned}
\sum_{i=1}^{m} M_{i, k} \leq & \sum_{i=1}^{m}\left(\alpha a_{k} C_{i}+(1-\alpha) b_{k} D_{i}\right)^{2}+m\left(\alpha a_{k} v_{k}+(1-\alpha) b_{k} \sigma_{k}\right)^{2} \\
& +2 \sum_{i=1}^{m}\left(\alpha a_{k} C_{i}+(1-\alpha) b_{k} D_{i}\right)\left(\alpha a_{k} \mu_{k}+(1-\alpha) b_{k} \tau_{k}\right) \\
& +2 \sum_{i=1}^{m}\left(\alpha a_{k} C_{i}+(1-\alpha) b_{k} D_{i}\right) \sum_{j=1}^{i-1}\left\{\alpha a_{k} C_{j}+(1-\alpha) b_{k} D_{j}\right. \\
& \left.+\alpha a_{k} v_{k}+(1-\alpha) b_{k} \sigma_{k}\right\}\left(\text { since } \mu_{k} \leq v_{k} \text { and } \tau_{k} \leq \sigma_{k}\right) \\
\leq & \sum_{i=1}^{m}\left(\alpha a_{k} C_{i}+(1-\alpha) b_{k} D_{i}+\alpha a_{k} v_{k}+(1-\alpha) b_{k} \sigma_{k}\right)^{2} \\
& +2 \sum_{i=1}^{m}\left(\alpha a_{k} C_{i}+(1-\alpha) b_{k} D_{i}+\alpha a_{k} v_{k}+(1-\alpha) b_{k} \sigma_{k}\right) \times \\
& \sum_{j=1}^{i-1}\left(\alpha a_{k} C_{j}+(1-\alpha) b_{k} D_{j}+\alpha a_{k} v_{k}+(1-\alpha) b_{k} \sigma_{k}\right) \\
= & \left(\sum_{i=1}^{m}\left(\alpha a_{k} C_{i}+(1-\alpha) b_{k} D_{i}+\alpha a_{k} v_{k}+(1-\alpha) b_{k} \sigma_{k}\right)\right)^{2} \\
= & \left(\alpha a_{k} C+(1-\alpha) b_{k} D+\alpha m a_{k} v_{k}+(1-\alpha) m b_{k} \sigma_{k}\right)^{2} .
\end{aligned}
$$

This completes the proof of Lemma 5.2.

\section{References}

Arrow, K., \& Hurwicz, L. (1957). Gradient methods for constrained maxima. Operations Research, 5(3), 258-265.

Ausubel, L. (2004). An efficient ascending-bid auction for multiple objects. American

Economic Review, 94(5), 1452-1475. 
Bertsekas, D. (2009). Convex Optimization Theory. Athena Scientific.

Bertsekas, D. (2012). Incremental gradient, subgradient, and proximal methods for convex optimization: A survey. In S. Nowozin, S. Sra, \& S. J. Wright (Eds.), Optimization for Machine Learning (pp. 1-38). Cambridge, MA: MIT Press.

Bikhchandani, S., \& Mamer, J. W. (1997). Competitive equilibrium in an exchange economy with indivisibilities. Journal of Economic Theory, 74, 384-413.

Chatterjee, K., \& Samuelson, W. (1983). Bargaining under incomplete information. Operations Research, 31, 835-851.

Chen, B., Fujishuge, S., \& Yang, Z. (2016). Random decentralized market processes for stable job matchings with competitive salaries. Journal of Economic Theory, $165,25-36$.

Clarke, F., Y. Ledyaev, R. S., \& Wolenski, P. (1988). Nonsmooth Analysis and Control Theory. New York: Springer.

Correa, R., \& Lemaréchal, C. (1993). Convergence of some algorithms for convex minimization. Mathematical Programming, 62, 261-275.

Fama, E. F. (1965). Random walks in stock-market prices. Financial Analysts Journal, 51(1), 75-80.

Friedman, D., \& Rust, J. (1993). The double auction market: Institutions, theories and evidence. Santa Fe Institute Studies in the Sciences of Complexity, XIV.

Fujishige, S., \& Yang, Z. (2003). A note on Kelso and Crawford's gross substitutes condition. Mathematics of Operations Research, 28(3), 463-469.

Gresik, T. (1991). The efficiency of linear equilibria of sealed-bid double auctions. Journal of Economic Theory, 53, 173-184.

Gul, F., \& Stacchetti, E. (1999). Walrasian equilibrium with gross substitutes. Journal of Economic Theory, 87, 9-24.

Gul, F., \& Stacchetti, E. (2000). The English auction with differentiated commodities. Journal of Economic Theory, 92, 66-95.

Hatfield, J. W., \& Kojima, F. (2010). Substitutes and stability for matching with contracts. Journal of Economic Theory, 145(5), 1704-1723.

Hatfield, J. W., \& Milgrom, P. (2005). Matching with contracts. American Economic Review, 95, 913-935.

Hayek, F. A. (1945). The use of knowledge in society. American Economic Review, 35, 519-530.

Jackson, M., \& Swinkels, J. (2005). Existence of equilibrium in single and double private value auctions. Econometrica, 73(1), 93-139.

Kelly, F., Maulloo, A., \& Tan, D. (1998). Rate control in communication networks: Shadow prices, proportional fairness, and stability. Journal of the Operational Research Society, 49, 237-252.

Kelso, A. S., \& Crawford, V. (1982). Job matching, coalition formation and gross 
substitute. Econometrica, 50, 1483-1504.

Kibardin, V. M. (1980). Decomposition into functions in the minimization problem. Automation and Remote Control, 40(9), 1311-1333.

Kojima, F., \& Yamashita, T. (2016). Double auction with interdependencet values: Incentives and efficiency. Theoretical Economics, forthcoming.

Ma, J. (1998). Competitive equilibrium with indivisibilities. Journal of Economic Theory, 82, 458-468.

Ma, J., \& Li, Q. (2011). Bubbles, crashes and efficiency with double auction mechanisms. Social Science Research Network Working paper, 2163435.

Ma, J., \& Nie, F. (2003). Walrasian equilibrium in an exchange economy with indivisibilities. Mathematical Social Sciences, 46, 159-192.

Milgrom, P. (2000). Putting auction theory to work. Journal of Political Economy, $\underline{108}(2), 245-272$.

Myerson, R. B., \& Satterthwaite, M. A. (1983). Efficient mechanisms for bilateral trading. Journal of Economic Theory, 29(2), 265-281.

Nedić, A., \& Bertsekas, D. P. (2001). Incremental subgradient methods for nondifferentiable optimization. SIAM Journal on Optimization, 12, 109-138.

Nedić, A., \& Ozdaglar, A. (2009). Approximate primal solutions and rate analysis for dual subgradient methods. SIAM Journal on Optimization, 19, 1757-1780.

Ram, S. S., Nedić, A., \& Veeravalli, V. V. (2009). Incremental stochastic subgradient algorithms for convex optimization. SIAM Journal on Optimization, 20, 691-717.

Satterthwaite, M. A., \& Williams, S. R. (1989). Bilateral trade with the sealed bid $k$-double auction: Existence and efficiency. Journal of Economics Theory, 48(1), 107-133.

Shiller, R. J. (1981). Do stock prices move too much to be justified by subsequent changes in dividends? American Economic Review, 71, 421-436.

Shleifer, A. (1999). Inefficient Markets: An Introduction to Behavioral Finance. Oxford: Oxford University Press.

Smith, A. (1776). The Wealth of Nations. New York: Random House, Modern Library Edition (this edition, 1937).

Smith, V. L. (1962). An experimental study of competitive market behavior. Journal of Political Economy, 70(2), 111-137.

Smith, V. L. (1965). Experimental auction markets and the Walrasian hypothesis. Journal of Political Economy, 73, 387-393.

Smith, V. L., Suchanek, G. L., \& Williams, A. A. (1988). Bubbles, crashes, and endogenous expectations in experimental spot asset markets. Econometrica, 56, 1119-1151.

Solodov, M. V., \& Zavriev, S. K. (1998). Error stability properties of generalized gradient-type algorithms. Journal of Optimization Theory and Applications, $\underline{98}$, 
663-680.

Sun, N., \& Yang, Z. (2006). Equilibria and indivisibilities: Gross substitutes and complements. Econometrica, 74, 1385-1402.

Sun, N., \& Yang, Z. (2008). On a class of discrete convolution concave functions and their applications. Yokohama Business Review, 29, 113-120.

Sun, N., \& Yang, Z. (2009). A double-track adjustment process for discrete markets with substitutes and complements. Econometrica, 77, 933-952.

Sun, N., \& Yang, Z. (2014). An efficient and incentive compatible dynamic auction for multiple complements. Journal of Political Economy, 122, 422-466.

Wilson, R. (1985). Incentive efficiency of double auctions. Econometrica, 53, 1101-1115.

Xia, M., Stallaert, J., \& Whinston, A. B. (2005). Solving the combinatorial double auction problem. European Journal of Operational Research, 164(1), 239-251.

Xu, X., Ma, J., \& Xie, X. (2014). Double auction mechanisms on Markovian networks. Expert Systems with Applications, 41(16), 7032-7045.

Xu, X., Ma, J., \& Xie, X. (2015). Convergences of price processes under a probabilistic double auction. Social Science Research Network Working Paper, 2684783.

$\mathrm{Xu}, \mathrm{X}$., Ma, J., \& Xie, X. (2016). Convergence of Markovian price processes in a financial market transaction model. Operational Research: An International Journal, forthcoming, 1-35. 